\title{
MicroRNAs expression profile in CCR6+ regulatory T cells
}

Backgroud. CCR6+ CD4+ regulatory T cells (CCR6+Tregs), a distinct Tregs subset, played an important role in various immune diseases. Recent evidence showed that microRNAs (miRNAs) are vital regulators in the function of immune cells. However, the potential role of miRNAs in the function of CCR6+Tregs remains largely unknown. In this study, we detected the expression profile of miRNAs in CCR6+ Tregs. Materials and Methods. The expression profile of miRNAs as well as genes in CCR6+Tregs or CCR6-Tregs from Balb/c mice were detected by microarray. The signaling pathways were analyzed using Keggs pathway library. Results. We found that there were 58 miRNAs significantly upregulated and 62 downregulated up to 2 fold in CCR6+Tregs compared with CCR6-Tregs. Moreover, 1391 genes were observed with 3 fold change and 20 signaling pathways were enriched using Keggs pathway library. Conclusion. The present data firstly showed CCR6+Tregs expressed specific miRNAs pattern, which provide an insight into the role of miRNAs in the biological function of distinct Tregs subsets. 
2 Juanjuan Zhao ${ }^{1 \#}$, Yongju $\mathrm{Li}^{1 \#}$, Yan $\mathrm{Hu}^{1}$, Chao Chen ${ }^{1}$, Ya Zhou ${ }^{2}$, Yijin Tao ${ }^{1}$, Mengmeng Guo , 3 Nalin $\mathrm{Qin}^{1}, \mathrm{Lin} \mathrm{Xu}^{1 *}$

4

$5{ }^{1}$ Department of Immunology, Zunyi Medical College, Guizhou, 563000 China

$6 \quad{ }^{2}$ Department of Medical physics, Zunyi Medical College, Guizhou, 563000 China

7

8

9

$10{ }^{\#}$ These authors contributed equally to this work.

$11{ }^{*}$ Correspondence to:

12 Dr. Lin Xu, Department of immunology, Zunyi Medical College, GuiZhou Province, 563003 P.R.China, Email:

13 xulinzhouya@163.com

14

15

16

17

18

19

20

21

22

23

24 
CC chemokine receptor type 6 (CCR6), a family member of chemokine receptor, was widely expressed in various immune cells (Duhen $\square$ Campbell, 2014; Paradis et al., 2014 ; Wong et al., 2013) The interaction of CCR6 and its distinct ligand CCL20 mediated the migration of immune cells into immune reaction sites (Chen et al., 2011; Kallal et al., 2010). Recent evidence showed that CCR6 also was functional expressed on $\mathrm{CD}^{+} \mathrm{CD} 25^{+}$regulatory $\mathrm{T}$ cells (Tregs) (Rivino et al., 2010). And $\mathrm{CCR} 6^{+}$subset of Tregs, displayed memory/effector phenotype, played an important role in various immune diseases (Kitamura et al., 2010). Such as, Kleinewietfeld et al reported that $\mathrm{CCR}^{+}$Tregs were involved in the pathogenesis of experimental allergic encephalomyelitis (EAE) (Kleinewietfeld et al., 2005). In the setting of tumors, Lamprecht et al reported that CCR6 ${ }^{+}$Tregs might favor immune escape of Hodgkin/Reed-Sternberg (HRS) cells (Lamprecht et al., 2008). Similarly, our recent work further showed that $\mathrm{CCR}^{+}{ }^{+}$subset of Treg cells were dominantly enriched in tumor mass and closely related to poor prognosis of breast cancer patients (Xu et al., 2010). Notably, the predominant proliferation triggered by DCs was critical for their enrichment and suppressive capacity in tumor mass $(X u$ et al., 2011). However, the exact regulation mechanism involved in the biological function including proliferation and suppressive capacity of this Tregs subset remains largely unknown, which might be helpful for the understanding of contribution of distinct Treg subsets to immunosuppression and ultimately aid the designing of therapy for clinical related disease.

MicroRNAs (miRNAs) are endogenous, non-coding single-stranded RNAs that are approximately 20 nucleotides in length, and have emerged as a key regulator in physiology as well as pathology attributable to its ability to downregulate gene expression through mRNA destabilization/degradation and translation repression by binding onto either 3' UTR of the target mRNA. Recent studies have shown that different immune cells expressed distinct miRNAs pattern and these miRNA molecules have the ability to modify the expression of target genes and subsequently regulate the function of immune cells (Johanson et al., 2014; Danger et al., 2014; Gigli $\square$ Maizon, 2013). Such as, miR-21 was highly expressed in $\mathrm{CD}^{+} \mathrm{T}$ cells (Sommers et al., 2013). And silencing of miR-21 could alter the proliferation and function of $\mathrm{CD}^{+}{ }^{+} \mathrm{T}$ cells (Wang et al., 2014). However, whether $\mathrm{CCR}^{+}$Tregs also expressed specific miRNAs pattern and the potential role of these miRNAs in the biological function of these cells remains to be elucidated.

To this end, in the present study, the expression pattern of miRNAs in the CCR6 $6^{+}$Tregs was evaluated.

54 Moreover, the mRNA expression profile which might be affected by these miRNAs also was investigated. Our 55 data showed that CCR6 ${ }^{+}$Tregs expressed distinct miRNAs signature, which associated with different expression of related genes. These finding might provided novel insight in the role of miRNAs in the function of distinct subset of Tregs.

58 


\section{MATERIAL AND METHODS}

\section{Animals}

62 Female Balb/c mice 5-6 weeks of age were purchased from the Center of Experimental Animal, Fudan

63 University (Shanghai, China). All animals were housed in the pathogen free mouse colony at our institution and

64 all animal experiments were performed according to the guidelines for the Care and Use of Laboratory Animals

65 (Ministry of Health, PR China, 1998) and all the experimental procedure was approved by the 66 ethical guidelines of Zunyi Medical College Laboratory Animal Care and Use Committee (No. 20130108).

\section{Flow cytometry}

Flow cytometry was performed on a FACSAria (BD Biosciences) with CellQuest Pro software using directly conjugated mAbs against the following human or murine markers: CD4-PerCP, CD25-allophycocyanin, and CCR6-FITC with corresponding isotype-matched controls (either BD Biosciences or eBioscience Systems). Foxp3 staining was conducted using the Murine Regulatory T cell staining kit (eBioscience) and run according

miRNA Microarray

All sample labeling and GeneChip procession were performed in Kangchen Biotech Corp (Guangzhou, China; http:// http://www.kangchen.com.cn/english). One microgram of total RNA was labeled and then hybridized to miRCURY LNA ${ }^{\mathrm{TM}}$ microRNA, 7.0 arrays for 16 hours at $48^{\circ} \mathrm{C}$. All washing steps were performed by a GeneChip Fluidics Station 450 and GeneChip were scanned with the GeneChip Scanner 3000 7G. Partek was used to determine ANOVA p-values and fold changes for miRNAs. Data accessible at NCBI GEO database (Xu L et al., 2014), accession GSE60041. Species annotations were added and used to filter only those miRNA found in Mus musculus.

\section{Gene Expression Microarray}

85 Total RNA was first converted to cDNA, followed by in vitro transcription to make cRNA. 5 ug of single 86 stranded cDNA was synthesized; end labeled and hybridized, for 16 hours at $45^{\circ} \mathrm{C}$, to Mouse Gene $1.0 \mathrm{ST}$

87 arrays. All washing steps were performed by a GeneChip Fluidics Station 450 and GeneChip were scanned 88 with the Axon GenePix 4000B microarray scanner. Partek was used to determine ANOVA p-values and fold 89 changes for genes.

\section{Real time PCR}

92 All reagents, primers, and probes were obtained from Applied Biosystems. A U6 endogenous control was used 93 for normalization. Reverse transcriptase reactions and real-time PCR were performed according to the 94 manufacturer's protocols (Applied Biosystems). RNA concentrations were determined with a NanoDrop 95 instrument (NanoDrop Technologies). One nanogram of RNA per sample was used for the assays. All RT 96 reactions, including no-template controls and RT minus controls, were run in triplicate in GeneAmp PCR 9700 
97 Thermocycler (Applied Biosystems). Gene expression levels were quantified using the ABI Prism 7900HT

98 sequence detection system (Applied Biosystems). Relative expression was calculated using the comparative 99 threshold cycle (Ct) method. The primers used for target genes: murine miR-142a (fwd):5`-

100 TGGCATGAGGATCAGCAGGG-3`, murine miR-142a (rev):5 '-GGCAGTCCGCAGCTCTAG- -G-3`; murine 101 miR-21 (fwd): 5'-GCGTGCTAATGGTGGA-3’, murine miR-21 (rev): 5'-CAGGCGTAT- -CAGTGGG-3’.

102

103 Statistical analyses

104 Statistical analyses of the data were performed with the aid of analysis programs in SPSS12.0 software.

105 Statistical evaluation was performed using one way analysis of variance (ANOVA) or $\mathrm{t}$ test using the program

106 PRISM 4.0 (GraphPad Software Inc., San Diego, CA, USA). The p values $<0.05$ were considered significant

107 and are indicated on the figures accompanying this article as follows unless otherwise indicated: $* p<0.05$.

108 Unless otherwise indicated, error bars represent SD. 


\section{RESULTS}

\section{MicroRNA expression profiles in $\mathbf{C C R 6}^{+}$Tregs}

111 Our previous data showed that $\mathrm{CCR}^{+}$Tregs could dominantly enriched in tumor mass, which was 112 associated with their potential proliferation activity compared with their CCR6 ${ }^{-}$counterpart (Xu et al., 2010; Xu 113 et al., 2011). In order to characterize the miRNA expression profile that regulates genes involved in potential 114 proliferation activity of $\mathrm{CCR}^{+}$Tregs, we performed a microarray assay using Affymatrix: GeneChip miRNA 1153.0 Array that contains 1111 mouse probe sequences. Microarray assays showed that miRNA were expressed 116 differentially in CCR6 $6^{+}$Tregs. A total of 120 miRNA were significantly altered with the criteria of 2.0 fold 117 change with $\mathrm{p}<0.05$ (Tab 1). Out of the 120 altered miRNAs, 58 were upregulated in CCR6 ${ }^{+}$Tregs compared 118 with CCR6-Tregs. As shown in apie graph of miRNA distribution based on their fold changes in expression 119 (Fig 1A), the majority of altered (88 out of 120) fell into the range of 2.0 to 4.0 fold up or downregulation.

120 Only eleven miRNAs (five up-regulated and another six down-regulated) displayed over 10 fold changes 121 between two groups (Fig 1B).

122 To further investigate which miRNAs was potentially involved in the proliferation activity of CCR ${ }^{+}{ }^{T}$ regs, 1236 miRNAs among 120 altered miRNAs, which was well documented related to the proliferation activity of T 124 cells, was showed (Fig 1C). In addition, we further confirmed the expression of miR-142a and miR-21 in these 1256 miRNAs by quantitative PCR. Data showed that the expression of miR-142a and miR-21 were also 126 significantly upregulated in CCR6 ${ }^{+}$Tregs compared with those in CCR6-Tregs respectively (Fig S1, p $<0.05$ ), 127 which was consistent with the data in miRNA array.

\section{Gene expression profile and signaling pathway in $\mathrm{CCR6}^{+}$Tregs}

To investigate the possible function of these altered expression miRNA molecules in CCR6 ${ }^{+}$Tregs, we detected the global gene expression changes in CCR6 ${ }^{+}$Tregs. CCR6 ${ }^{+}$Tregs and CCR6-Tregs were harvested and subjected to gen expression microarray assay. As shown in fig 2a, the altered gene expression profiles in 133 CCR6 $^{+}$Tregs as shown in a heat map. Given a three-fold change and $\mathrm{p}<0.05$ (up and down) in differential expression as a cut-off, the number of altered genes was reduced to 1391; 651 of them were downregulated, and 740 genes were up regulated (Table 2 and Table 3 ).

136 To clarify which signaling pathways were altered in CCR6 ${ }^{+}$Tregs, we applied the KEGG library and 137 performed enrichment analysis for microarray data. Twenty signaling pathways were enriched with the criteria 138 of 2 fold changes (Table 4), which include the inositol phosphate metabolism, $\mathrm{T}$ cell receptor signaling 139 pathway, phosphatidylinositol signaling system, mTOR signaling pathway, primary immunodeficiency and 140 some cancer signaling pathway. Some genes from those pathways were downregulated or upregulated, Such as 141 in T cell receptor signaling pathway, ICOS, ZAP70, LAT, PLC- $\gamma 1$, ITK, Ras and p38 were downregulated (Fig 142 3). The mTOR pathway evenly consisted of both up and downregulated genes, in which RSK, STRAD and 143 Raptor were downregulated and PIK3c2b, TSC1 and MO25 were upregulated (Tab 2 and 3). 


\section{DISCUSSION}

145 Previous studies have indicated that $\mathrm{CD} 4{ }^{+} \mathrm{CD} 25^{+}$regulatory $\mathrm{T}$ cells (Treg) were a heterogeneous cell 146 population comprising different subsets that play distinct roles in diverse animal models or human clinical 147 disease, mediating immune suppression or immune tolerance (Pankratz et al., 2014; Erfani et al., 2014; Lee et 148 al., 2014). Thus, the investigation involved in regulation of function of distinct subset of Tregs is valuable.

149 Recent evidence showed that $\mathrm{CCR}^{+}$subsets of Tregs played an important role in various immune responses. 150 Such as Villares et al reported that $\mathrm{CCR} 6^{+}$Tregs could inhibit the function of pathological CD $4{ }^{+} \mathrm{Th} 1$ cells 151 mediated EAE pathology (Villares et al., 2009). We also found that CCR6 ${ }^{+}$Tregs, but not their CCR6152 counterpart, could dominantly enriched in tumor mass and potential inhibited the function of effector T cells in 153 vivo (Xu et al., 2010; Xu et., 2011). These finding might support the fact that CCR6 ${ }^{+}$subset of Tregs played a 154 critical role in tumor immunity. However, the regulation mechanism involved in the function of this subset 155 Tregs remains largely unknown. Recent studies provided some clues to solve this problem since they showed 156 that miRNAs may play a regulatory role in the development and function of Tregs (Smigielska-Czepiel et al., 157 2014; Fayyad-Kazan et al., 2012). To gain new insight into the role of miRNAs in the function of CCR6 ${ }^{+}$Tregs, 158 differentially expressed miRNAs were investigated by microarray assay. Moreover, the regulatory pathways of 159 putative target genes were also analyzed in CCR6 ${ }^{+}$Tregs. It was found that there were significantly different 160 miRNA expression patterns in CCR6 ${ }^{+}$Tregs and CCR6-Tregs. The difference could be described one handred 161 and twenty miRNAs, including 58 up- and 62 down-regulated, had more than 2-fold differential expression 162 between CCR6 ${ }^{+}$Tregs and CCR6-Tregs. We speculated that the above two differences might be a reason for the different function such as proliferation activity of CCR6 ${ }^{+}$Tregs compared with CCR6 ${ }^{-}$Tregs.

miR-142, a distinct member of miRNAs family, is highly conserved across species and is linked to chromosome 3p22.3/12q14. Recent evidence showed that miR-142 was highly expressed in Tregs and could regulate the expansion of Tregs in response to stimulation (Zhou et al., 2013). In this study, we observed that miR-142 was significantly upregulated in CCR6 $6^{+}$Tregs. Predicated by TargetScan and FINDTAR3, 14 genes were putative target of miR-142, in which 4 genes was downregulated (Fig S2). We also noticed that Gfi1 was downregulated in CCR6 ${ }^{+}$Tregs, indicating Gfil might be a target of miR-142. Supportively, Shi et al demonstrated that Gfil was critical for the development of Tregs. Moreover, loss of Gfi-1 could endow the aberrant expansion of Tregs through IL-2 signaling pathway (Shi et al., 2013). Thus, further study on miR-142

172 function will help us understand the regulatory role of miR-142 in the function and proliferation of 173 CCR6 $^{+}$Tregs.

174 MiR-21 is one of the first discovered miRNAs that is known to be widespread in human tissues such as heart, 175 lung, brain and liver. Accumulating data highlighted that miR-21 can regulate the biological character of 176 various cells including survival, invasion and apoptosis (Shi et al., 2013; Bullock et al., 2013; Niu et al., 2012). 177 Especially, miR-21 was documented as an important regulator actor in the proliferation of cells. For example, 178 Liu et al reported that miR-21 could enhance the proliferation of cancer cells through Akt pathway (Liu et al., 179 2014). Interestingly, recent evidences further showed that miR-21 was also functional expressed in $\mathrm{T}$ cells 180 (Sommers et al., 2013). Such as miR-21 could support survival of CD4 ${ }^{+} \mathrm{T}$ cells and was an important signature 
181 in $\mathrm{CD}^{+} \mathrm{T}$ cells proliferation. And silencing of miR-21 could alter the proportion of $\mathrm{CD} 4^{+} \mathrm{T}$ cells in lupus mice 182 (Wang et al., 2014). Consistently, we observed an increase in the expression of miR-21 in CCR6 ${ }^{+}$Tregs.

183 Therefore, further study on the possible role of miR-21 also was valuable for the understanding of proliferation 184 of CCR6 $6^{+}$Tregs.

185 The data from gene expression microarray showed that 1391 genes (651 downregulated and 740 up186 regulated) were significantly changed with more than three fold in CCR6 ${ }^{+}$Tregs. Among them, some genes 187 have been demonstrated to be involved in the proliferation and function of Tregs. For example, TCR signaling 188 pathway was critical for the proliferation and function of CCR6 ${ }^{+}$Tregs. We noticed that there were some genes 189 such as ZAP70, LAT and PLC-1 was downregulated, indicating weak transduction of TCR signaling pathway 190 in CCR6 ${ }^{+}$Tregs. Consistently, previous literatures showed that CCR6 ${ }^{+}$Tregs displayed a memory/effector 191 phenotype and more sensitivity for the stimulation of TCR (Kleinewietfeld et al., 2005). In addition, Hanschen 192 et al reported that TCR stimulation could induce rapid and higher activation of ZAP70 in Tregs (Hanschen et 193 al., 2012), indicating that phosphorylation of ZAP70 also might be important for the proliferation of 194 CCR6 $6^{+}$Tregs. Therefore, these altered genes might be good targets for the proliferation and function of 195 CCR6 $^{+}$Tregs in successive research work. In addition, we would point out that we did not find any prominently 196 annotated biological category using miRNA-mRNA anti-correlations in present study. We proposal it reflect the

197 fact that the interaction of miRNA and mRNA in the biology of CCR6 $6^{+}$Tregs is complex, which remains to be 198 exactly elucidated in next work.

199 In summary, to our knowledge, it is the first time to show that CCR6 ${ }^{+}$Tregs, a distinct subset of Tregs, 200 expressed distinct miRNA profile, which will help us to understand the potential role miRNAs in the biological 201 function of CCR6 $6^{+}$Tregs.

\section{AUTHOR CONTRIBUTIONS}

206 Juang J. Zhao and Yong J. Li performed the experiments, analyzed the data, wrote the paper; Yan Hu and Chao 207 Chen performed the experiments, analyzed the data; Ya Zhou, Yi J Tao and Meng M Guo performed the 208 experiments; Na L Qin and Dan Tian wrote the paper; Lin Xu conceived and designed the experiments, analyzed the data, wrote the paper.

\section{SUPPLEMENTAL INFORMATION}

213 Supplemental figure 1. The relative expression of miR-142a and miR-21 in CCR6 ${ }^{+}$Tregs.

214 Supplemental figure 2. The expression of putative targets of miR-142a in CCR6 ${ }^{+}$Tregs. 
Bullock MD, Pickard KM, Nielsen BS, Sayan AE, Jenei V, Mellone M, Mitter R, Primrose JN, Thomas GJ, Packham GK, Mirnezami AH. 2013. Pleiotropic actions of miR-21 highlight the critical role of deregulated stromal microRNAs during colorectal cancer progression. Cell Death \& Disease 4: e684.

Chen KJ, Lin SZ, Zhou L, Xie HY, Zhou WH, Taki-Eldin A, Zheng SS. 2011. Selective recruitment of regulatory $\mathrm{T}$ cell through CCR6-CCL20 in hepatocellular carcinoma fosters tumor progression and predicts poor prognosis. PLoS One 6: e24671.

Danger R, Braza F, Giral M, Soulillou JP, Brouard S. 2014. MicroRNAs, major players in B cells homeostasis and function. Frontiers in Immunology 5: 98.

Duhen T, Campbell DJ. 2014. IL-1 $\beta$ Promotes the Differentiation of Polyfunctional Human CCR6+ CXCR3+ Th1/17 Cells That Are Specific for Pathogenic and Commensal Microbes. Journal Immunology 193:120129.

Erfani N, Hamedi-Shahraki M, Rezaeifard S, Haghshenas M, Rasouli M, Samsami Dehaghani A. 2014. Foxp3 $+\mathrm{T}$ Cells in Peripheral Blood of Patients with Epithelial Ovarian Cancer. Iranian Journal of Immunology 11: 105-112.

Fayyad-Kazan H, Rouas R, Fayyad-Kazan M, Badran R, El Zein N, Lewalle P, Najar M, Hamade E, Jebbawi F, Merimi M, Romero P, Burny A, Badran B, Martiat P. 2012. MicroRNA profile of circulating CD4-positive regulatory $\mathrm{T}$ cells in human adults and impact of differentially expressed microRNAs on expression of two genes essential to their function. Journal of Biological Chemistry 287: 9910-9922.

Gigli I, Maizon DO. 2013. microRNAs and the mammary gland: a new understanding of gene expression. Genetics and Molecular Biology 36: 465-474.

Hanschen M, Tajima G, O'Leary F, Hoang K, Ikeda K, Lederer JA. 2012. Phospho-flow cytometry based analysis of differences in T cell receptor signaling between regulatory T cells and CD4+ T cells. Journal of Immunological Methods 376: 1-12.

Johanson TM, Skinner JP, Kumar A, Zhan Y, Lew AM, Chong MM. 2014. The role of microRNAs in lymphopoiesis. International Journal of Hematology [Epub ahead of print]

Kallal LE, Schaller MA, Lindell DM, Lira SA, Lukacs NW. 2010. CCL20/CCR6 blockade enhances immunity to RSV by impairing recruitment of DC. European Journal of Immunology 40: 1042-1052.

Kitamura K, Farber J M, Kelsall B L. 2010. CCR6 Marks Regulatory T Cells as a Colon-Tropic, IL-10Producing Phenotype. Journal of Immunol 185: 3295-3304.

Kleinewietfeld M, Puentes F, Borsellino G, Battistini L, Rötzschke O, Falk K. 2005. CCR6 expression defines regulatory effector/memory-like cells within the CD25+ CD4+ T-cell subset. Blood 105: 28772886.

Lamprecht B, Kreher S, Anagnostopoulos I, Jöhrens K, Monteleone G, Jundt F, Stein H, Janz M, Dörken B, Mathas S. 2008. Aberrant expression of the Th2 cytokine IL-21 in Hodgkin lymphoma cells regulates STAT3 signaling and attracts Treg cells via regulation of MIP-3 $\alpha$. Blood 112: 3339-3347.

Lee KM, Stott RT, Zhao G, SooHoo J, Xiong W, Lian MM, Fitzgerald L, Shi S, Akrawi E, Lei J, Deng S, Yeh H, Markmann JF, Kim JI. 2014. TGF- $\beta$-producing regulatory B cells induce regulatory T cells and promote transplantation tolerance. European Journal of Immunology 44: 1728-1736.

Liu Y, Nie H, Zhang K, Ma D, Yang G, Zheng Z, Liu K, Yu B, Zhai C, Yang S. 2014. A feedback regulatory loop between HIF-1 $\alpha$ and miR-21 in response to hypoxia in cardiomyocytes. FEBS letters [Epub ahead of print].

Niu J, Shi Y, Tan G, Yang CH, Fan M, Pfeffer LM, Wu ZH. 2012. DNA damage induces NF-кB-dependent microRNA-21 up-regulation and promotes breast cancer cell invasion. Journal of Biological Chemistry 287: 21783-21795.

Pankratz S, Bittner S, Herrmann AM, Schuhmann MK, Ruck T, Meuth SG, Wiendl H. 2014. Human CD4+ HLA-G+ regulatory T cells are potent suppressors of graft-versus-host disease in vivo. The FASEB Journal [Epub ahead of print].

Paradis M, Mindt BC, Duerr CU, Rojas OL, Ng D, Boulianne B, McCarthy DD, Yu MD, Summers deLuca LE, Ward LA, Waldron JB, Philpott DJ, Gommerman JL, Fritz JH. 2014. A TNF- $\alpha-C C L 20-$ CCR6 Axis Regulates Nod1-Induced B Cell Responses. Journal Immunology 192: 2787-2799.

Rivino L, Gruarin P, Häringer B, Steinfelder S, Lozza L, Steckel B, Weick A, Sugliano E, Jarrossay D, Kühl AA, Loddenkemper C, Abrignani S, Sallusto F, Lanzavecchia A, Geginat J. 2010. CCR6 is expressed on an IL-10-producing, autoreactive memory $\mathrm{T}$ cell population with context-dependent 
regulatory function. Journal of Experimental Medicine 207: 565-577.

Shi LZ, Kalupahana NS, Turnis ME, Neale G, Hock H, Vignali DA, Chi H. 2013. Inhibitory role of the transcription repressor Gfil in the generation of thymus-derived regulatory T cells. Proceedings of the National Academy of Sciences of the United States of America 110: E3198-3205.

Shi Z, Zhang J, Qian X, Han L, Zhang K, Chen L, Liu J, Ren Y, Yang M, Zhang A, Pu P, Kang C. 2013. AC1MMYR2, an inhibitor of dicer-mediated biogenesis of oncomir miR-21, reverses epithelialmesenchymal transition and suppresses tumor growth and progression. Cancer Research 73: 5519-5531.

Smigielska-Czepiel K, van den Berg A, Jellema P, van der Lei RJ, Bijzet J, Kluiver J, Boots AM, Brouwer E, Kroesen BJ. 2014. Comprehensive analysis of miRNA expression in T-cell subsets of rheumatoid arthritis patients reveals defined signatures of naive and memory Tregs. Genes \& Immunity 15: 115-125.

Sommers CL, Rouquette-Jazdanian AK, Robles AI, Kortum RL, Merrill RK, Li W, Nath N, Wohlfert E, Sixt KM, Belkaid Y, Samelson LE. 2013. miRNA signature of mouse helper T cell hyper-proliferation. PLoS One 8: e66709.

Villares R, Cadenas V, Lozano M, Almonacid L, Zaballos A, Martínez-A C, Varona R. 2009. CCR6 regulates EAE pathogenesis by controlling regulatory CD4+ T-cell recruitment to target tissues. European Journal of Immunology 39:1671-1681.

Wang L, He L, Zhang R, Liu X, Ren Y, Liu Z, Zhang X, Cheng W, Hua ZC. 2014. Regulation of T lymphocyte activation by microRNA-21. Molecular Immunology 59: 163-171.

Wong EB, Akilimali NA, Govender P, Sullivan ZA, Cosgrove C, Pillay M, Lewinsohn DM, Bishai WR, Walker BD, Ndung'u T, Klenerman P, Kasprowicz VO. 2013. Low Levels of Peripheral CD161+ CD8+ Mucosal Associated Invariant T (MAIT) Cells Are Found in HIV and HIV/TB Co-Infection. PLoS One 8: e83474.

Xu L, Xu W, Qiu S, Xiong S. 2010. Enrichment of CCR6Foxp3 regulatory T cells in the tumor mass correlates with impaired CD8 T cell function and poor prognosis of breast cancer. Clinical Immunology 135: 466475.

Xu L, Xu W, Wen Z, Xiong S. 2011. In situ prior proliferation of CD4+ CCR6+ regulatory T cells facilitated by TGF- $\beta$ secreting DCs is crucial for their enrichment and suppression in tumor immunity. PloS one $\mathbf{6}$ : e20282.

Zhou Q, Haupt S, Prots I, Thümmler K, Kremmer E, Lipsky PE, Schulze-Koops H, Skapenko A. 2013. miR-142-3p is involved in CD25+ CD4 T cell proliferation by targeting the expression of glycoprotein A repetitions predominant. Journal of Immunology 190: 6579-6788. 


\section{Table 1 (on next page)}

120 miRNAs altered in CCR6 ${ }^{+}$Tregs 
Table 1. 120 miRNAs altered in $\mathrm{CCR6}^{+}$Tregs.

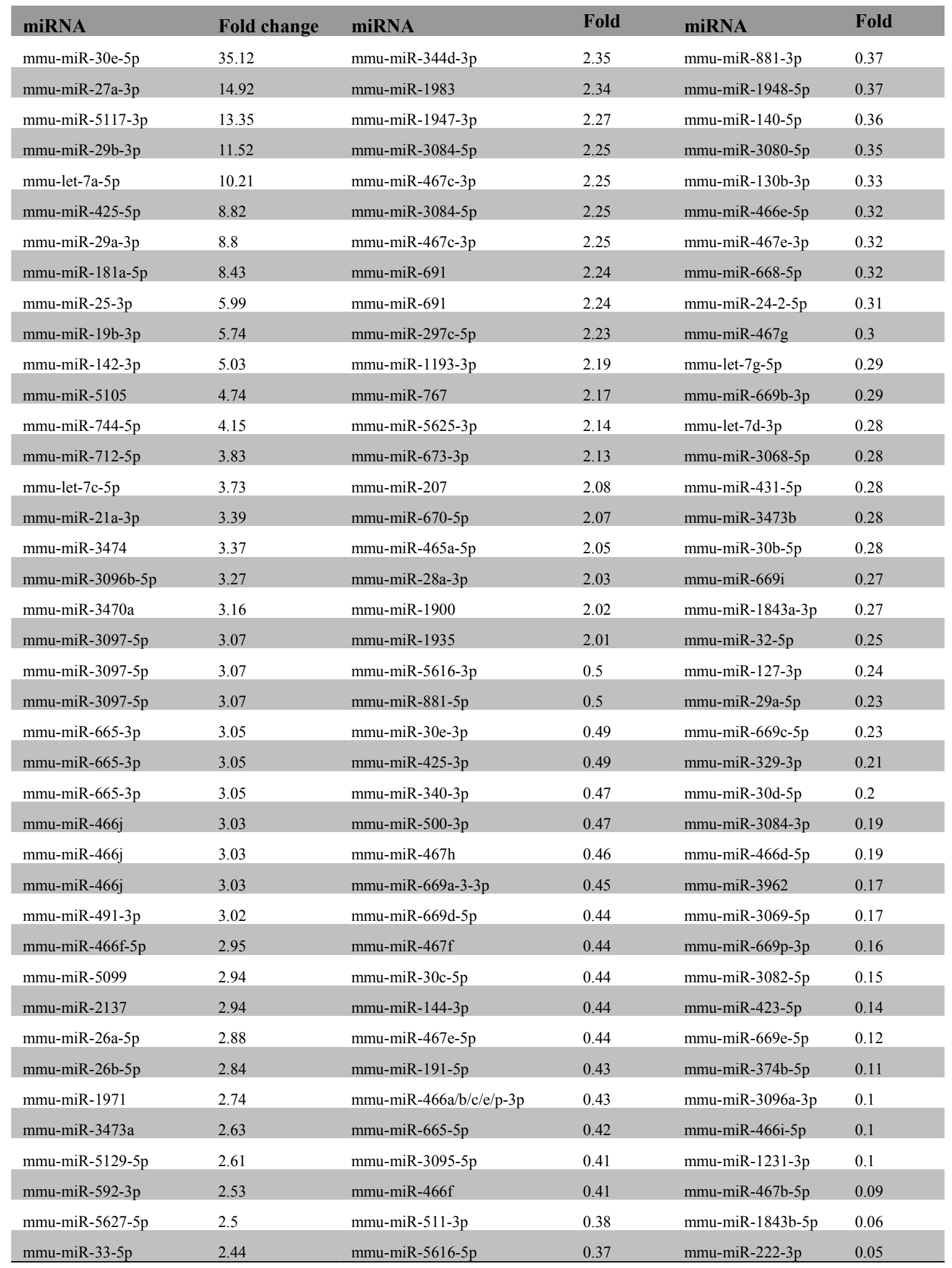




\section{Table 2 (on next page)}

Over 3-fold up-regulation genes (651)in CCR6+Tregs 
Table2. Over 3-fold up-regulation genes (651)in CCR6 ${ }^{+}$Tregs

\begin{tabular}{|c|c|c|c|c|c|}
\hline Target gene & Fold & Target gene & Fold & Target gene & Fold \\
\hline Kenh7 & 21.32 & Aurkb & 3.94 & Fam195b & 3.33 \\
\hline Olfr250 & 16.83 & Faim3 & 3.93 & Sept11 & 3.32 \\
\hline Gm11623 & 13.12 & AU022751 & 3.93 & Chi313 & 3.32 \\
\hline Treml4 & 12.86 & Igh & 3.92 & Adora3 & 3.32 \\
\hline Den & 11.30 & Gda & 3.92 & Tcf712 & 3.32 \\
\hline Gm13766 & 10.31 & Olfr777 & 3.92 & Sdc1 & 3.32 \\
\hline Rpap1 & 9.22 & Gm4698 & 3.91 & Cecr2 & 3.32 \\
\hline V1rc16 & 9.14 & Wdr66 & 3.91 & A2ld 1 & 3.32 \\
\hline Dlgap5 & 9.12 & S100a16 & 3.91 & Haаo & 3.32 \\
\hline N/A & 8.35 & $\mathrm{Cd} 22$ & 3.91 & AW146020 & 3.32 \\
\hline Cts6 & 8.32 & 2610035F20Rik & 3.91 & Veph1 & 3.32 \\
\hline Atp6v1b1 & 8.24 & Igh & 3.91 & N/A & 3.32 \\
\hline Dnahc12 & 8.24 & Kiss1 & 3.91 & Dhdds & 3.31 \\
\hline Adam29 & 8.17 & Brdt & 3.90 & $\mathrm{H} 2-\mathrm{Ab} 1$ & 3.31 \\
\hline AW551984 & 8.11 & Pycrl & 3.89 & N/A & 3.31 \\
\hline Hven1 & 8.04 & Gm2987 & 3.89 & Vmn2r38 & 3.31 \\
\hline D630033O11Rik & 7.87 & Igh & 3.88 & 2010204K13Rik & 3.31 \\
\hline 4933405L10Rik & 7.59 & Npepps & 3.88 & $\mathrm{Cd} 72$ & 3.31 \\
\hline Igh & 7.50 & Clip2 & 3.87 & Gzmb & 3.30 \\
\hline Fam131b & 7.23 & Gm3758 & 3.87 & Zfp385b & 3.30 \\
\hline Ly6g5b & 7.15 & Gys1 & 3.87 & Pdgfra & 3.30 \\
\hline Klra13 & 6.71 & N/A & 3.87 & 1700057H15Rik & 3.30 \\
\hline Neurod6 & 6.62 & Xrcc1 & 3.87 & Txnde17 & 3.29 \\
\hline $\operatorname{Mef} 2 \mathrm{c}$ & 6.62 & N/A & 3.86 & Etv5 & 3.29 \\
\hline P2ry4 & 6.43 & Zfp553 & 3.85 & Fcerlg & 3.29 \\
\hline Neil3 & 6.39 & Nol9 & 3.85 & Gm14920 & 3.29 \\
\hline Trappc2 & 6.39 & Tsen54 & 3.85 & $\mathrm{Olfm} 4$ & 3.29 \\
\hline Tmem109 & 6.38 & Ints7 & 3.85 & N/A & 3.29 \\
\hline Clec4n & 6.38 & Tcfeb & 3.85 & Erall & 3.28 \\
\hline Vmn2r102 & 6.36 & P2ryl & 3.84 & 2310030N02Rik & 3.28 \\
\hline N/A & 6.36 & Hist1h2bg & 3.84 & Gm13403 & 3.28 \\
\hline Gm10649 & 6.32 & Mxd1 & 3.84 & Idh3b & 3.28 \\
\hline Cage1 & 6.31 & Cadps2 & 3.83 & Chd5 & 3.28 \\
\hline Gtf2ird2 & 6.28 & N/A & 3.83 & Tssk2 & 3.28 \\
\hline $\mathrm{N} / \mathrm{A}$ & 6.27 & 2310061I04Rik & 3.83 & Cbwd1 & 3.28 \\
\hline Eyal & 6.22 & Fcer2a & 3.82 & Robol & 3.27 \\
\hline Mpo & 6.09 & Klhl13 & 3.82 & Whsc1 & 3.27 \\
\hline Gpr152 & 6.07 & Pah & 3.82 & Bmp1 & 3.27 \\
\hline AI324046 & 6.04 & Zdhhc3 & 3.81 & Pygl & 3.27 \\
\hline Ccdc82 & 5.99 & Lcn2 & 3.81 & Pvrl4 & 3.27 \\
\hline 4933411K16Rik & 5.98 & Zbtb34 & 3.81 & $\mathrm{Cd} 180$ & 3.27 \\
\hline Pigt & 5.96 & Sirpb1 & 3.81 & Tpsg1 & 3.26 \\
\hline Havcr2 & 5.94 & Adam1a & 3.79 & Gprc5a & 3.26 \\
\hline 4933402D24Rik & 5.92 & Ace2 & 3.79 & Gm13375 & 3.26 \\
\hline Myom1 & 5.92 & C86187 & 3.79 & 1810034E14Rik & 3.26 \\
\hline Kif2c & 5.72 & March4 & 3.79 & Illb & 3.26 \\
\hline
\end{tabular}




\begin{tabular}{|c|c|c|c|c|c|}
\hline Olfr514 & 5.65 & Pigq & 3.79 & C330016O10Rik & 3.26 \\
\hline Gm7306 & 5.62 & Lingo1 & 3.78 & Ank2 & 3.25 \\
\hline Dnajc28 & 5.59 & Nuak2 & 3.78 & Ins2 & 3.25 \\
\hline 4930578G10Rik & 5.58 & $\mathrm{~V} 1 \mathrm{rd} 2$ & 3.77 & Hrh4 & 3.25 \\
\hline 4930517G24Rik & 5.57 & Igh & 3.77 & Trp53rk & 3.25 \\
\hline Gm12260 & 5.57 & $\operatorname{Cdc} 20$ & 3.77 & Grik1 & 3.24 \\
\hline N/A & 5.53 & Adam9 & 3.76 & Asgr1 & 3.24 \\
\hline Gm2847 & 5.53 & Gm13152 & 3.76 & Lrrc59 & 3.24 \\
\hline Gp49a & 5.50 & Cenf & 3.76 & $\mathrm{~N} / \mathrm{A}$ & 3.24 \\
\hline Fcamr & 5.49 & Csgalnact2 & 3.76 & 2810408A11Rik & 3.24 \\
\hline Klhdc7b & 5.48 & Vps53 & 3.75 & Gcet2 & 3.24 \\
\hline Cacnalf & 5.46 & Uggt2 & 3.74 & Lrrk2 & 3.24 \\
\hline 4930467D21Rik & 5.42 & Rbm8a & 3.73 & Pira11 & 3.24 \\
\hline Masp1 & 5.34 & Igk & 3.73 & Tusc1 & 3.24 \\
\hline N/A & 5.32 & Pcbp1 & 3.73 & Usp35 & 3.24 \\
\hline Stk33 & 5.32 & Klk15 & 3.73 & Panx3 & 3.24 \\
\hline Xirp1 & 5.31 & Smox & 3.73 & Vtila & 3.23 \\
\hline Prune & 5.30 & Gm5393 & 3.73 & Nudt1611 & 3.23 \\
\hline Brpfl & 5.27 & Txnl4b & 3.72 & Tnk1 & 3.23 \\
\hline Zdbf2 & 5.26 & 9130017N09Rik & 3.72 & Ighv14-2 & 3.23 \\
\hline 4930432E11Rik & 5.24 & Rims1 & 3.72 & Hspb11 & 3.23 \\
\hline Arhgap24 & 5.24 & Spire1 & 3.71 & Blk & 3.23 \\
\hline N/A & 5.22 & $\mathrm{~N} / \mathrm{A}$ & 3.71 & Zdhhc4 & 3.23 \\
\hline Il15 & 5.20 & Psmg4 & 3.70 & Phka1 & 3.22 \\
\hline Plin1 & 5.18 & Mrps36 & 3.70 & Micalcl & 3.22 \\
\hline Spink10 & 5.18 & Pstk & 3.70 & Gm13089 & 3.22 \\
\hline Snca & 5.15 & Trmt2a & 3.70 & RP23-480B19.10 & 3.22 \\
\hline Styxl1 & 5.14 & Nsg2 & 3.70 & Rwdd3 & 3.22 \\
\hline Ranbp17 & 5.14 & Anxa1 & 3.70 & 1110037F02Rik & 3.22 \\
\hline Mcam & 5.09 & Lpcat2 & 3.69 & Krtap13 & 3.21 \\
\hline Vmn2r121 & 5.09 & Asb4 & 3.69 & $\mathrm{Cd} 22$ & 3.21 \\
\hline Chi314 & 5.08 & Sprr2a3 & 3.69 & Hist1h2ab & 3.21 \\
\hline Ltb4r2 & 5.02 & Rps6kb1 & 3.69 & 2700008G24Rik & 3.21 \\
\hline Ppp1r3d & 5.02 & Zfp282 & 3.68 & N/A & 3.21 \\
\hline Gm2705 & 5.00 & Wdfy4 & 3.68 & Chst14 & 3.21 \\
\hline Et14 & 4.98 & Gm2448 & 3.68 & $\mathrm{~A} 2 \mathrm{bp} 1$ & 3.20 \\
\hline Fam108b & 4.93 & Lta $4 \mathrm{~h}$ & 3.67 & Gm2739 & 3.20 \\
\hline Adamts8 & 4.92 & 1600020E01Rik & 3.67 & Lman1 & 3.20 \\
\hline Akr1c13 & 4.91 & Psg29 & 3.66 & Timp1 & 3.20 \\
\hline Gm11543 & 4.89 & Sik3 & 3.66 & $\operatorname{Rad} 54 b$ & 3.20 \\
\hline $\mathrm{I} 117 \mathrm{c}$ & 4.89 & 4933421E11Rik & 3.65 & 1700012C08Rik & 3.20 \\
\hline Ccdc30 & 4.89 & Ltf & 3.65 & LOC668727 & 3.20 \\
\hline Tmed9 & 4.88 & Lpp & 3.65 & Syt13 & 3.20 \\
\hline Fam46a & 4.87 & $\mathrm{H} 2-\mathrm{Aa}$ & 3.65 & Zfp710 & 3.19 \\
\hline N/A & 4.87 & Gm2586 & 3.64 & Pex11b & 3.19 \\
\hline Clic5 & 4.86 & Lphn3 & 3.64 & Ncf1 & 3.19 \\
\hline Gm5153 & 4.85 & A530023O14Rik & 3.64 & Sh3pxd2a & 3.19 \\
\hline Fzd1 & 4.84 & Msh5 & 3.64 & Ush2a & 3.19 \\
\hline
\end{tabular}




\begin{tabular}{|c|c|c|c|c|c|}
\hline Hemt1 & 4.82 & Gm11981 & 3.64 & Trim29 & 3.19 \\
\hline Anxa1 & 4.79 & Crem & 3.64 & Pecam1 & 3.18 \\
\hline Retnlg & 4.78 & Lmo2 & 3.63 & Mtus1 & 3.18 \\
\hline Gm7219 & 4.77 & Gm4846 & 3.63 & Fam55b & 3.17 \\
\hline Tmem63b & 4.77 & Apoo & 3.63 & Gm2461 & 3.17 \\
\hline Clec $4 d$ & 4.75 & Btbd7 & 3.63 & Golim4 & 3.17 \\
\hline 4933416M06Rik & 4.73 & Med8 & 3.62 & Acp1 & 3.17 \\
\hline Zyx & 4.73 & Mgl1 & 3.62 & $\mathrm{Gm} 2695$ & 3.17 \\
\hline Klk1b4 & 4.72 & Med31 & 3.62 & Kdelc2 & 3.17 \\
\hline Defb30 & 4.71 & Abcal6 & 3.61 & Myo1c & 3.17 \\
\hline Insc & 4.65 & Hes6 & 3.61 & Gprc5b & 3.16 \\
\hline Hs3st2 & 4.65 & Igh & 3.61 & $\operatorname{Ren} 3$ & 3.16 \\
\hline Ubap1 & 4.62 & Cdkl5 & 3.60 & Rassf4 & 3.16 \\
\hline Gpr56 & 4.61 & Oxgr1 & 3.60 & Adrb2 & 3.16 \\
\hline Igh-VJ558 & 4.61 & F5 & 3.60 & $\mathrm{Cd} 36$ & 3.16 \\
\hline Igh & 4.61 & Psmd13 & 3.59 & Slc34a3 & 3.15 \\
\hline Cpne2 & 4.61 & Clock & 3.59 & $A \cot 4$ & 3.15 \\
\hline 2610028H24Rik & 4.60 & Stab1 & 3.58 & Ccdc157 & 3.15 \\
\hline Ras110a & 4.58 & Coasy & 3.58 & Igl-V1 & 3.15 \\
\hline Mrpl33 & 4.58 & Fcrla & 3.57 & 4930534B04Rik & 3.15 \\
\hline Fn3k & 4.58 & Cybb & 3.56 & Gm6127 & 3.15 \\
\hline 9430025M13Rik & 4.57 & D2hgdh & 3.56 & 3110056O03Rik & 3.15 \\
\hline Gm13083 & 4.55 & Igh & 3.56 & Kenb2 & 3.15 \\
\hline Klra6 & 4.54 & Adamtsl1 & 3.56 & Atp8b4 & 3.15 \\
\hline 4933412E24Rik & 4.53 & $\mathrm{BC} 005705$ & 3.56 & Gm10883 & 3.15 \\
\hline Zfp707 & 4.52 & Loxl4 & 3.56 & Bcr & 3.14 \\
\hline Rapgefl1 & 4.52 & Ncapd2 & 3.55 & Mtus1 & 3.14 \\
\hline Scyl2 & 4.50 & $\mathrm{Hdc}$ & 3.55 & Sgsm3 & 3.14 \\
\hline Rab711 & 4.49 & Gem & 3.55 & Tdp1 & 3.14 \\
\hline Scfd1 & 4.49 & N/A & 3.55 & Tcf15 & 3.14 \\
\hline N/A & 4.48 & Sepx1 & 3.55 & Lmbr1 & 3.14 \\
\hline Gm4395 & 4.48 & Tubgcp5 & 3.54 & Ermap & 3.14 \\
\hline Odf4 & 4.46 & Cpne2 & 3.54 & 2210009G21Rik & 3.14 \\
\hline Nfam1 & 4.46 & Rarres1 & 3.54 & N/A & 3.13 \\
\hline Topbp1 & 4.46 & Ebf3 & 3.54 & Dhx35 & 3.13 \\
\hline Grhl1 & 4.46 & Csflr & 3.54 & El13 & 3.13 \\
\hline Guf1 & 4.45 & N/A & 3.54 & 4930406D18Rik & 3.13 \\
\hline Trpm3 & 4.44 & Igh & 3.54 & Ubd & 3.13 \\
\hline Ciita & 4.43 & $\mathrm{~N} / \mathrm{A}$ & 3.54 & Gm6425 & 3.13 \\
\hline Hist1h2ak & 4.42 & Mfsd3 & 3.53 & Hist $1 \mathrm{~h} 3 \mathrm{e}$ & 3.13 \\
\hline Igh & 4.42 & Homer2 & 3.53 & Slc22a17 & 3.13 \\
\hline Fcgr $2 b$ & 4.42 & Zbtb16 & 3.53 & Serpinb1c & 3.12 \\
\hline Wac & 4.42 & Ifltd 1 & 3.52 & $\operatorname{Sin}$ & 3.12 \\
\hline Msmb & 4.41 & Gm10693 & 3.52 & Gm10766 & 3.12 \\
\hline Plac8 & 4.41 & Ptgs1 & 3.52 & Adipor1 & 3.12 \\
\hline Nr5al & 4.38 & Sh $2 d 3 c$ & 3.51 & Gm684 & 3.11 \\
\hline Gm13446 & 4.37 & V1rc29 & 3.51 & Il1f9 & 3.11 \\
\hline Vmn2r73 & 4.37 & Lrp1 & 3.51 & Kcnj16 & 3.10 \\
\hline
\end{tabular}




\begin{tabular}{|c|c|c|c|c|c|}
\hline Pfkfb4 & 4.37 & Nova1 & 3.51 & Car1 & 3.10 \\
\hline Phyhipl & 4.36 & N/A & 3.51 & Psme4 & 3.10 \\
\hline Gpatch4 & 4.36 & 4930578N18Rik & 3.49 & Siglec5 & 3.10 \\
\hline Cenph & 4.36 & A030001D20Rik & 3.49 & N/A & 3.10 \\
\hline Gm13154 & 4.35 & Hsf4 & 3.49 & Igk-C & 3.10 \\
\hline $\operatorname{Tm} 2 \mathrm{~d} 1$ & 4.35 & Trem3 & 3.49 & N/A & 3.10 \\
\hline Ptplad2 & 4.35 & Arhgap24 & 3.48 & Igh & 3.10 \\
\hline Gm13597 & 4.34 & Lins2 & 3.48 & 2310002J15Rik & 3.09 \\
\hline Nkd1 & 4.34 & Igh & 3.48 & G630018N14Rik & 3.09 \\
\hline Phox $2 b$ & 4.33 & Prnd & 3.48 & $\mathrm{Rbx} 1$ & 3.09 \\
\hline Cyp2j7 & 4.33 & 4930529M08Rik & 3.47 & Gm8787 & 3.08 \\
\hline Pstpip2 & 4.31 & 3110009E18Rik & 3.47 & N/A & 3.08 \\
\hline Fam81b & 4.29 & Hist1h2bb & 3.46 & $\mathrm{Gm} 7170$ & 3.08 \\
\hline Pira3 & 4.29 & Ncapg & 3.46 & $\mathrm{Cd} 19$ & 3.08 \\
\hline Gpr112 & 4.28 & E030019B13Rik & 3.46 & Wfdc1 & 3.08 \\
\hline 5031414D18Rik & 4.27 & Gm3528 & 3.46 & Casp12 & 3.08 \\
\hline Trpm3 & 4.27 & Gm15498 & 3.46 & 6330416G13Rik & 3.07 \\
\hline Slco4cl & 4.27 & Cryz & 3.46 & Il6ra & 3.07 \\
\hline Zfp354b & 4.25 & Stard4 & 3.46 & Scd1 & 3.07 \\
\hline Camp & 4.24 & Bfsp2 & 3.45 & H2afy & 3.07 \\
\hline Ric3 & 4.24 & Rpap1 & 3.45 & Lmbrd1 & 3.07 \\
\hline Tsfm & 4.23 & Vsig1 & 3.44 & Piral & 3.07 \\
\hline Abcc3 & 4.22 & Olfr1431 & 3.44 & Gm5468 & 3.07 \\
\hline $\mathrm{BC} 035044$ & 4.22 & Abcb4 & 3.44 & Pgap1 & 3.07 \\
\hline C230096C10Rik & 4.22 & Vwc2 & 3.44 & Prom2 & 3.07 \\
\hline Nkg7 & 4.20 & Rpap1 & 3.44 & Nubp1 & 3.07 \\
\hline Gm15623 & 4.20 & 5830477G23Rik & 3.43 & $\mathrm{C} 1 \mathrm{qb}$ & 3.07 \\
\hline Casc1 & 4.20 & Gypa & 3.43 & Tcf712 & 3.06 \\
\hline Lsm1 & 4.19 & Slc25a42 & 3.43 & Ebfl & 3.06 \\
\hline Anxa6 & 4.19 & Arhgap26 & 3.43 & Itgb6 & 3.06 \\
\hline D130009I18Rik & 4.17 & $\mathrm{Ccl} 6$ & 3.42 & Terf2 & 3.06 \\
\hline Illb & 4.17 & Cbfa2t3 & 3.42 & Prosc & 3.06 \\
\hline Pcdh17 & 4.16 & Snx29 & 3.42 & $\mathrm{~N} / \mathrm{A}$ & 3.06 \\
\hline Clec4d & 4.16 & Ube $2 w$ & 3.42 & I19r & 3.05 \\
\hline Alk & 4.16 & Slc1a1 & 3.41 & Gm14206 & 3.05 \\
\hline $\mathrm{Cd} 79 \mathrm{~b}$ & 4.15 & Olfr399 & 3.41 & Fignl1 & 3.05 \\
\hline $\mathrm{Zc} 3 \mathrm{~h} 7 \mathrm{~b}$ & 4.15 & D930016D06Rik & 3.41 & Dhrs3 & 3.05 \\
\hline $\mathrm{Mc} 4 \mathrm{r}$ & 4.15 & Hs2st1 & 3.41 & Ikbkg & 3.05 \\
\hline Sept8 & 4.13 & Pou3f3 & 3.41 & Map3k7ip1 & 3.05 \\
\hline Gp49a & 4.13 & Ccdc46 & 3.41 & Lcat & 3.05 \\
\hline N/A & 4.12 & Olfr1434 & 3.41 & Itsn1 & 3.05 \\
\hline Smarcd1 & 4.12 & Pcdh15 & 3.40 & Creld1 & 3.05 \\
\hline 2700050L05Rik & 4.11 & N/A & 3.40 & Gm9121 & 3.04 \\
\hline Fmn12 & 4.11 & Ctbp2 & 3.40 & Klrb1c & 3.04 \\
\hline Gm11686 & 4.11 & $\mathrm{Pla} 2 \mathrm{~g} 7$ & 3.40 & Gpr116 & 3.04 \\
\hline Ubely1 & 4.10 & Clk2 & 3.40 & Igh-6 & 3.04 \\
\hline 1600012P17Rik & 4.10 & Gen1 & 3.40 & Igk-C & 3.04 \\
\hline Irf5 & 4.09 & Stoml1 & 3.39 & Cstf1 & 3.04 \\
\hline
\end{tabular}




\begin{tabular}{|c|c|c|c|c|c|}
\hline Caskin 1 & 4.08 & Prpf19 & 3.39 & Cel & 3.04 \\
\hline Cd3001f & 4.08 & Acer2 & 3.39 & Slc30a1 & 3.04 \\
\hline Oosp1 & 4.07 & Rhox $2 \mathrm{c}$ & 3.39 & N/A & 3.04 \\
\hline $\mathrm{X} \operatorname{lr} 3 \mathrm{a}$ & 4.07 & Snn & 3.38 & Gm10193 & 3.03 \\
\hline Nol4 & 4.07 & V1rb8 & 3.38 & Gm9880 & 3.03 \\
\hline Map2k7 & 4.06 & Sema4a & 3.38 & N/A & 3.03 \\
\hline Gm5577 & 4.05 & Tmeffl & 3.38 & $\mathrm{Gm} 2436$ & 3.03 \\
\hline Trmt12 & 4.04 & Olfr395 & 3.38 & Prr14 & 3.03 \\
\hline Sec1411 & 4.04 & LOC677563 & 3.38 & Spsb1 & 3.03 \\
\hline D930015E06Rik & 4.03 & $\operatorname{Rfc} 2$ & 3.37 & $\mathrm{Hbb}-\mathrm{b} 2$ & 3.03 \\
\hline Slpi & 4.03 & A430075N02 & 3.37 & Acrv1 & 3.02 \\
\hline Ggal & 4.03 & Pvrl2 & 3.36 & Shmt1 & 3.02 \\
\hline Tex101 & 4.03 & Snx8 & 3.36 & Bcl11a & 3.02 \\
\hline Itsn1 & 4.02 & Adamts1 & 3.36 & N/A & 3.02 \\
\hline Gm3323 & 4.02 & Pnmt & 3.36 & Ly6g & 3.02 \\
\hline Gm2954 & 4.01 & Poll & 3.36 & $\mathrm{Cd} 74$ & 3.02 \\
\hline Slc $35 \mathrm{e} 4$ & 4.01 & Serpinalf & 3.35 & Fchsd2 & 3.02 \\
\hline Clqa & 4.00 & $\mathrm{Pla} 2 \mathrm{~g} 12 \mathrm{a}$ & 3.35 & Pik3cg & 3.02 \\
\hline Retnlg & 4.00 & Kel & 3.35 & 3300005D01Rik & 3.01 \\
\hline $\mathrm{Cul2}$ & 3.99 & Cks2 & 3.35 & Prc1 & 3.01 \\
\hline Plekhm3 & 3.99 & Axl & 3.35 & Hyou1 & 3.01 \\
\hline Cyth2 & 3.98 & 2010110P09Rik & 3.34 & Gnb2 & 3.01 \\
\hline $\operatorname{Scfd} 2$ & 3.98 & Spink12 & 3.34 & Pla2g15 & 3.01 \\
\hline Gns & 3.98 & 4933400N17Rik & 3.33 & 2010308F09Rik & 3.01 \\
\hline Yifla & 3.96 & Cd3001f & 3.33 & Gm10270 & 3.00 \\
\hline N/A & 3.95 & Hist1h4f & 3.33 & Pak7 & 3.00 \\
\hline N/A & 3.95 & Zfp800 & 3.33 & C730027P07Rik & 3.00 \\
\hline
\end{tabular}




\section{Table 3 (on next page)}

Over 3-fold down-regulation genes (740) in CCR6+ Tregs 
Table3. Over 3-fold down-regulation genes (740) in $\mathrm{CCR6}^{+}$Tregs

\begin{tabular}{|c|c|c|c|c|c|}
\hline Target gene & Fold change & Target gene & Fold & Target gene & Fold \\
\hline Il2ra & 25.65 & Atxn711 & 4.27 & Trim37 & 3.41 \\
\hline N/A & 18.99 & $\mathrm{Gm} 5282$ & 4.25 & Ksr2 & 3.41 \\
\hline Gm9119 & 15.47 & St3gal3 & 4.25 & ENSMUSG00000079376 & 3.41 \\
\hline Il2ra & 15.05 & 4930417O13Rik & 4.24 & Ptpn5 & 3.40 \\
\hline N/A & 14.55 & Trerfl & 4.24 & 9230117E06Rik & 3.40 \\
\hline Ctla4 & 14.24 & Klk6 & 4.23 & N/A & 3.40 \\
\hline Gal3st1 & 12.37 & 2610042L04Rik & 4.22 & N/A & 3.40 \\
\hline $\mathrm{Gm} 3453$ & 12.21 & Cyp4f41-ps & 4.22 & Plekhal & 3.39 \\
\hline Gal & 12.20 & Clcn 1 & 4.21 & Trav3n-3 & 3.39 \\
\hline ENSMUSG00000072735 & 11.93 & Abcb7 & 4.20 & Lrsam1 & 3.39 \\
\hline Foxp3 & 11.69 & $\operatorname{Bcs} 11$ & 4.20 & Olfr109 & 3.39 \\
\hline Cyb5r2 & 11.65 & Stk19 & 4.18 & Rsbn1 & 3.39 \\
\hline Phkg1 & 10.53 & Sectmla & 4.18 & Odf1 & 3.39 \\
\hline Ikzf2 & 10.44 & Fmrlnb & 4.17 & $\mathrm{Mc} 2 \mathrm{r}$ & 3.38 \\
\hline Evc2 & 10.17 & Pnkd & 4.17 & Ifna6 & 3.38 \\
\hline $\mathrm{Il17rc}$ & 10.00 & N/A & 4.17 & Gm7223 & 3.38 \\
\hline Plekhg5 & 9.93 & Gpr110 & 4.17 & Cntn4 & 3.38 \\
\hline ENSMUSG00000072735 & 9.66 & Inpp4b & 4.17 & N/A & 3.38 \\
\hline Acer2 & 9.56 & Gats13 & 4.17 & Gm10228 & 3.38 \\
\hline Neb & 9.55 & Dapk1 & 4.16 & Gm5169 & 3.37 \\
\hline Gpr45 & 9.49 & Gm3455 & 4.15 & R3hcc1 & 3.37 \\
\hline D15Wsu169e & 9.47 & Gm14717 & 4.14 & Slc38a1 & 3.37 \\
\hline Brunol5 & 9.44 & 1700001E04Rik & 4.14 & Inpp4b & 3.37 \\
\hline Pxdn & 9.44 & Pde4a & 4.13 & Nphp3 & 3.37 \\
\hline Gpr83 & 9.43 & $\operatorname{Slc} 35 \mathrm{f} 2$ & 4.13 & Csnk1g1 & 3.36 \\
\hline ENSMUSG00000072735 & 9.43 & Adam6b & 4.13 & Jazf1 & 3.36 \\
\hline Gm3727 & 9.36 & Penk & 4.13 & Arhgdig & 3.36 \\
\hline Gm3727 & 9.25 & 2510048L02Rik & 4.13 & Etaa1 & 3.36 \\
\hline N/A & 9.24 & Casp3 & 4.12 & $\mathrm{Cul} 2$ & 3.36 \\
\hline Gm11744 & 9.05 & Dcaf17 & 4.12 & Gm10837 & 3.36 \\
\hline Gm3339 & 8.66 & $\mathrm{Gm} 3182$ & 4.12 & Ppp2r3a & 3.36 \\
\hline Dpy1912 & 8.60 & 1500015O10Rik & 4.11 & Gm1574 & 3.35 \\
\hline Caskin2 & 8.31 & Acsl4 & 4.11 & Tspan 12 & 3.35 \\
\hline Ikzf2 & 8.22 & $\operatorname{Ddx} 43$ & 4.10 & Magi3 & 3.35 \\
\hline Tubgcp5 & 8.17 & AI987944 & 4.09 & 1110059M19Rik & 3.35 \\
\hline Gm2974 & 8.16 & Plin1 & 4.09 & Cpsf41 & 3.34 \\
\hline C230088H06Rik & 8.08 & Tox & 4.09 & Parp4 & 3.34 \\
\hline Fbxw27 & 8.05 & Gm10338 & 4.07 & Galr3 & 3.34 \\
\hline Gm14005 & 8.04 & Zscan12 & 4.06 & Adam33 & 3.34 \\
\hline Gm8362 & 7.95 & Fam71e1 & 4.06 & Frs3 & 3.33 \\
\hline Gm8297 & 7.93 & $\mathrm{Neb}$ & 4.06 & Ptgdr & 3.33 \\
\hline Pla2g2d & 7.93 & 100039441 & 4.05 & BE691133 & 3.33 \\
\hline Slc22a12 & 7.91 & $\mathrm{BC} 106179$ & 4.05 & Brp441 & 3.33 \\
\hline N/A & 7.87 & N/A & 4.05 & Gm11468 & 3.33 \\
\hline Cadm3 & 7.81 & Stab1 & 4.04 & Detn4 & 3.33 \\
\hline
\end{tabular}

PeerJ reviewing PDF | (v2014:07:2417:1:2:NEW 19 Aug 2014) 


\begin{tabular}{|c|c|c|c|c|c|}
\hline Cyhr1 & 7.58 & Tnfsf13b & 4.04 & E330021D16Rik & 3.33 \\
\hline B630019K06Rik & 7.58 & Mdfi & 4.03 & Gm3764 & 3.32 \\
\hline Inpp4b & 7.50 & A930002C04Rik & 4.03 & Cd300lg & 3.32 \\
\hline Ctla4 & 7.49 & Slc23a3 & 4.03 & Atg2a & 3.32 \\
\hline Сур2u1 & 7.47 & Col6a3 & 4.02 & Ankrd9 & 3.32 \\
\hline Gm3182 & 7.44 & Ghrh & 4.01 & Gm7225 & 3.32 \\
\hline Tgfb2 & 7.43 & A930017M01Rik & 4.01 & Pnpla7 & 3.32 \\
\hline Vwce & 7.41 & Itih51 & 4.01 & $\mathrm{Cd} 96$ & 3.31 \\
\hline LOC100036568 & 7.32 & Aurkc & 4.00 & 4833422F24Rik & 3.31 \\
\hline 1700029I01Rik & 7.31 & Itga6 & 4.00 & Thnsl2 & 3.31 \\
\hline Olfr701 & 7.29 & Mfrp & 3.99 & Pdcd11 & 3.31 \\
\hline Rfc3 & 7.29 & 1700042G15Rik & 3.99 & Robo4 & 3.31 \\
\hline Gm10014 & 7.22 & Mageh1 & 3.98 & Aven & 3.31 \\
\hline N/A & 7.20 & Ptpn13 & 3.98 & 1700026L06Rik & 3.31 \\
\hline LOC100038847 & 7.16 & Olfr227 & 3.98 & Lrig2 & 3.31 \\
\hline 544988 & 7.09 & 1700028M03Rik & 3.98 & Ehbp1 & 3.31 \\
\hline Gm4489 & 7.07 & Gpatch4 & 3.98 & Kctd9 & 3.30 \\
\hline LOC100038847 & 6.95 & Pxmp2 & 3.97 & Zbtb37 & 3.30 \\
\hline Nlrx1 & 6.94 & Mllt3 & 3.97 & Lrrc34 & 3.30 \\
\hline N/A & 6.92 & Gm10250 & 3.97 & Zfp30 & 3.30 \\
\hline Gm3642 & 6.92 & Cux1 & 3.96 & Ano2 & 3.29 \\
\hline Tgm1 & 6.90 & Csmd1 & 3.96 & N/A & 3.29 \\
\hline Dmd & 6.88 & Ptger3 & 3.96 & Tmem134 & 3.29 \\
\hline Foxp3 & 6.85 & Gm3990 & 3.95 & Sh2d6 & 3.29 \\
\hline ENSMUSG00000072735 & 6.82 & 2010005J08Rik & 3.94 & Olfr78 & 3.29 \\
\hline Gria1 & 6.82 & Olfr623 & 3.94 & Mapk8 & 3.29 \\
\hline Arhgef15 & 6.81 & ENSMUSG00000072735 & 3.94 & Upp1 & 3.29 \\
\hline Gm2888 & 6.79 & March7 & 3.94 & Gm2046 & 3.29 \\
\hline Fdft1 & 6.73 & N/A & 3.94 & Tex21 & 3.28 \\
\hline Gm3642 & 6.72 & Slc9a3 & 3.93 & Tnfrsf4 & 3.28 \\
\hline Nck2 & 6.70 & Rbm9 & 3.93 & Nol11 & 3.28 \\
\hline Adamts14 & 6.64 & Dtwd1 & 3.93 & 1700092C10Rik & 3.28 \\
\hline Zfp142 & 6.60 & C77370 & 3.93 & Gm3916 & 3.28 \\
\hline Gm3269 & 6.59 & $\mathrm{~N} / \mathrm{A}$ & 3.92 & Dmxl2 & 3.28 \\
\hline Gm3411 & 6.56 & Fbxw13 & 3.92 & ENSMUSG00000072735 & 3.28 \\
\hline 544988 & 6.53 & Amz2 & 3.92 & ENSMUSG00000079376 & 3.27 \\
\hline 9630058J23Rik & 6.53 & Nsl1 & 3.92 & 4930587E11Rik & 3.27 \\
\hline 2010109N18Rik & 6.51 & Plxna3 & 3.92 & Plcl1 & 3.27 \\
\hline N/A & 6.51 & Ppme1 & 3.90 & Srgap3 & 3.27 \\
\hline Brap & 6.51 & Gcgr & 3.90 & Prss39 & 3.27 \\
\hline Tmem210 & 6.47 & Sgcd & 3.90 & Dapk3 & 3.26 \\
\hline 4930486G11Rik & 6.46 & $\mathrm{~N} / \mathrm{A}$ & 3.89 & Fbxw24 & 3.26 \\
\hline Vmn2r46 & 6.46 & ENSMUSG00000068790 & 3.89 & Gm3626 & 3.26 \\
\hline 1110017D15Rik & 6.46 & Olfr658 & 3.88 & Mtap4 & 3.26 \\
\hline N/A & 6.43 & Fbxo15 & 3.88 & Gm3253 & 3.25 \\
\hline $\mathrm{N} / \mathrm{A}$ & 6.43 & Mrgprb4 & 3.88 & Cypt6 & 3.25 \\
\hline N/A & 6.38 & Ncoa7 & 3.87 & Aatf & 3.25 \\
\hline Gm3518 & 6.36 & Grin1 & 3.87 & $\mathrm{I} 12 \mathrm{rb}$ & 3.25 \\
\hline C430002F04Rik & 6.26 & 4933400A11Rik & 3.87 & Fam160a1 & 3.24 \\
\hline
\end{tabular}

PeerJ reviewing PDF | (v2014:07:2417:1:2:NEW 19 Aug 2014) 


\begin{tabular}{|c|c|c|c|c|c|}
\hline Gm13620 & 6.23 & Vmn2r72 & 3.87 & Ece1 & 3.24 \\
\hline Gm3685 & 6.23 & Pfkp & 3.87 & Nkx2-6 & 3.24 \\
\hline Zscan 10 & 6.22 & Igl & 3.86 & Pik3r2 & 3.24 \\
\hline Gm10340 & 6.22 & 4930445K14Rik & 3.86 & Slc25a21 & 3.24 \\
\hline Gm3159 & 6.21 & Krtap5-4 & 3.85 & Ptgfrn & 3.24 \\
\hline 5830403L16Rik & 6.16 & $\mathrm{Gm} 3424$ & 3.85 & N/A & 3.24 \\
\hline Gm3127 & 6.15 & $\mathrm{Cd} 247$ & 3.85 & Tbcel & 3.23 \\
\hline B930046C15Rik & 6.13 & Samsn 1 & 3.85 & Sgip1 & 3.23 \\
\hline Syne2 & 6.10 & Uty & 3.84 & 1700023L04Rik & 3.23 \\
\hline Gm3029 & 6.10 & Galk1 & 3.84 & Catsper3 & 3.23 \\
\hline 1600002D24Rik & 6.06 & 1700029G01Rik & 3.84 & Dgka & 3.23 \\
\hline $\mathrm{Gm} 2224$ & 6.04 & Agrn & 3.83 & 4930433N12Rik & 3.23 \\
\hline Gm4801 & 6.00 & Lrig2 & 3.83 & Rdh16 & 3.22 \\
\hline N/A & 5.97 & Slc25a27 & 3.83 & BC011248 & 3.22 \\
\hline Pitpnc1 & 5.94 & Gjb4 & 3.83 & Dlgap1 & 3.22 \\
\hline $\mathrm{Gm} 3476$ & 5.94 & Rgs16 & 3.83 & Olfr 1283 & 3.22 \\
\hline 6430562O15Rik & 5.92 & Cntn1 & 3.82 & Osbpl3 & 3.22 \\
\hline Vmn2r66 & 5.92 & Fndc7 & 3.82 & Foxi2 & 3.21 \\
\hline Gm3029 & 5.91 & Itk & 3.82 & Fam186a & 3.20 \\
\hline Gm3115 & 5.89 & N/A & 3.82 & Gm8356 & 3.20 \\
\hline Ndp & 5.84 & $\mathrm{H} 1 \mathrm{fx}$ & 3.81 & Timp4 & 3.20 \\
\hline Zfp329 & 5.83 & Pik3c2a & 3.80 & Tbc1d 8 & 3.20 \\
\hline Gpr64 & 5.82 & Ctsj & 3.80 & Srd5a1 & 3.20 \\
\hline Nav2 & 5.81 & Emid1 & 3.80 & Olfr242 & 3.20 \\
\hline Aven & 5.80 & Serpinale & 3.79 & Sel11 & 3.19 \\
\hline D030054H15Rik & 5.79 & Gm1330 & 3.79 & Mbnl2 & 3.19 \\
\hline Grik5 & 5.76 & Tbc1d4 & 3.79 & Plac9 & 3.19 \\
\hline Sgsm3 & 5.71 & Hs 1bp3 & 3.79 & N/A & 3.19 \\
\hline Ovol2 & 5.70 & Olfr961 & 3.79 & Slc12a1 & 3.19 \\
\hline Mc1r & 5.65 & Pask & 3.78 & Zfp169 & 3.19 \\
\hline Gm10371 & 5.65 & $\mathrm{BC} 060267$ & 3.78 & Dok7 & 3.18 \\
\hline N/A & 5.62 & Kpna1 & 3.78 & $\mathrm{Gm} 2275$ & 3.18 \\
\hline Luzp2 & 5.60 & Arg1 & 3.77 & $\mathrm{Gm} 2643$ & 3.18 \\
\hline Pthlh & 5.59 & Cts8 & 3.77 & Dpep2 & 3.18 \\
\hline 1700021F07Rik & 5.58 & Suclg1 & 3.77 & Pard6b & 3.18 \\
\hline Ccbel & 5.56 & 1700001E04Rik & 3.77 & Cyp17a1 & 3.17 \\
\hline $\mathrm{Cul7}$ & 5.55 & Synpo2 & 3.77 & 9330111N05Rik & 3.17 \\
\hline Cpped1 & 5.55 & 6030458C11Rik & 3.77 & Ccdc33 & 3.17 \\
\hline Fmnl3 & 5.54 & 1190002H23Rik & 3.77 & Tub & 3.17 \\
\hline D6Wsu163e & 5.53 & Rpusd3 & 3.76 & Rpl711 & 3.17 \\
\hline Serpinb9d & 5.49 & Gm6710 & 3.76 & AW495222 & 3.17 \\
\hline E030025P04Rik & 5.49 & Ikbkap & 3.76 & Ipcef1 & 3.17 \\
\hline Skap1 & 5.49 & N/A & 3.76 & Tle2 & 3.17 \\
\hline D0H4S114 & 5.47 & 4921523L03Rik & 3.75 & B3galnt2 & 3.17 \\
\hline Piwil2 & 5.45 & Speer4f & 3.75 & Ndel1 & 3.16 \\
\hline 4930524L23Rik & 5.45 & $\mathrm{Gm} 3047$ & 3.75 & Atp6v1c2 & 3.16 \\
\hline Ykt6 & 5.43 & Synpr & 3.74 & Hnrpll & 3.16 \\
\hline $\operatorname{Slc} 24 \mathrm{a} 3$ & 5.40 & N/A & 3.73 & Prrg1 & 3.16 \\
\hline
\end{tabular}




\begin{tabular}{|c|c|c|c|c|c|}
\hline N/A & 5.39 & 9030624G23Rik & 3.72 & Сур2j13 & 3.16 \\
\hline Gm6337 & 5.38 & Trp53inp2 & 3.72 & Espn & 3.16 \\
\hline Gm3149 & 5.34 & 9130401M01Rik & 3.72 & Mup1 & 3.15 \\
\hline Tnfrsf9 & 5.29 & Myst4 & 3.72 & Ptprr & 3.15 \\
\hline Ttn & 5.28 & Gm12836 & 3.72 & Snx11 & 3.15 \\
\hline Gpr52 & 5.27 & 2810039B14Rik & 3.71 & Chchd8 & 3.15 \\
\hline Cntfr & 5.26 & Fastk & 3.71 & Dnml & 3.15 \\
\hline ENSMUSG00000079376 & 5.25 & Inpp4b & 3.71 & Tbcld 25 & 3.15 \\
\hline LOC100038847 & 5.22 & N/A & 3.70 & Olfr1120 & 3.14 \\
\hline Mybpc2 & 5.22 & Prlh & 3.70 & Gm3981 & 3.14 \\
\hline Cdon & 5.16 & Mcm8 & 3.70 & Morc2a & 3.14 \\
\hline Slitrk6 & 5.16 & Gm15340 & 3.70 & Tt1l7 & 3.14 \\
\hline Dom3z & 5.14 & Gm4926 & 3.69 & Irf6 & 3.14 \\
\hline Gm3149 & 5.14 & Ebpl & 3.69 & A830039H05Rik & 3.13 \\
\hline Gm16521 & 5.14 & $\mathrm{~N} / \mathrm{A}$ & 3.69 & 1700024B18Rik & 3.13 \\
\hline Smarcall & 5.14 & 4930417O13Rik & 3.68 & Trap1a & 3.13 \\
\hline C230099D08Rik & 5.13 & Pcdh 15 & 3.68 & Vmn2r10 & 3.13 \\
\hline Olfr 1252 & 5.11 & Ctla4 & 3.68 & Nrn1 & 3.13 \\
\hline 4930599N23Rik & 5.11 & 4933432I09Rik & 3.68 & Mapkapk3 & 3.13 \\
\hline Gm3642 & 5.10 & Hsd $17 \mathrm{~b} 2$ & 3.68 & 3110082J24Rik & 3.13 \\
\hline Gm5634 & 5.09 & Fbp1 & 3.67 & Ccde65 & 3.13 \\
\hline Cngb1 & 5.08 & Gm5795 & 3.66 & Spag6 & 3.13 \\
\hline Pax3 & 5.07 & Gm8159 & 3.66 & AI428936 & 3.12 \\
\hline ENSMUSG00000068790 & 5.07 & Atf7 & 3.66 & Tiam1 & 3.12 \\
\hline 4632404H12Rik & 5.05 & $\mathrm{Kdm} 4 \mathrm{a}$ & 3.66 & Cenpk & 3.12 \\
\hline Vill & 5.03 & Ocrl & 3.66 & Rapsn & 3.12 \\
\hline Gm8050 & 5.02 & Sgoll & 3.65 & $\operatorname{Tm} 2 \mathrm{~d} 3$ & 3.11 \\
\hline Anks $4 b$ & 5.00 & Prox2 & 3.65 & Tiam1 & 3.11 \\
\hline St3gal6 & 4.96 & $\operatorname{Rnf26}$ & 3.65 & Tle2 & 3.11 \\
\hline 1700034I23Rik & 4.96 & N/A & 3.64 & Wbp11 & 3.11 \\
\hline Gm3172 & 4.95 & Bub1 & 3.64 & Olfr1128 & 3.11 \\
\hline Spata18 & 4.93 & Trim63 & 3.63 & Art1 & 3.11 \\
\hline Plcg1 & 4.92 & Slc6a9 & 3.62 & Grin3a & 3.11 \\
\hline Has2as & 4.91 & Dst & 3.61 & 1700110K17Rik & 3.10 \\
\hline Ntn4 & 4.90 & AI428936 & 3.61 & Bcat1 & 3.10 \\
\hline Skap1 & 4.88 & Marveld2 & 3.60 & Iigp1 & 3.10 \\
\hline Сyp2c50 & 4.88 & Esrrb & 3.60 & $\mathrm{Pla} 2 \mathrm{~g} 4 \mathrm{e}$ & 3.10 \\
\hline Cope & 4.87 & Gm4699 & 3.60 & Rpusdl & 3.10 \\
\hline N/A & 4.86 & Ttyh1 & 3.59 & Olfr638 & 3.10 \\
\hline Gm3642 & 4.86 & Rgs 16 & 3.59 & Agbl2 & 3.10 \\
\hline Tnfrsf18 & 4.85 & 8030463A06Rik & 3.59 & 4921509009Rik & 3.09 \\
\hline N/A & 4.82 & 4930578E11Rik & 3.58 & Olfr389 & 3.09 \\
\hline Snhg11 & 4.81 & Cacnb2 & 3.57 & Pcsk4 & 3.09 \\
\hline Gm6121 & 4.81 & Setd3 & 3.57 & Pou2f1 & 3.09 \\
\hline Ncoa7 & 4.80 & N/A & 3.57 & Brcc3 & 3.09 \\
\hline 1700025M24Rik & 4.75 & Gm2957 & 3.56 & Gm3034 & 3.09 \\
\hline S100a7a & 4.75 & Magea3 & 3.56 & Gm8362 & 3.09 \\
\hline Olfr140 & 4.74 & Syngr3 & 3.56 & D030028A08Rik & 3.09 \\
\hline
\end{tabular}




\begin{tabular}{|c|c|c|c|c|c|}
\hline Crem & 4.73 & Gm3127 & 3.55 & Fam118b & 3.08 \\
\hline Gab3 & 4.72 & ENSMUSG00000068790 & 3.55 & Cedc126 & 3.08 \\
\hline Ift80 & 4.71 & Tmem176a & 3.55 & Fbxw4 & 3.08 \\
\hline Secisbp2 & 4.69 & 1700081N11Rik & 3.55 & Cish & 3.08 \\
\hline 1110019B22Rik & 4.67 & $\mathrm{~N} / \mathrm{A}$ & 3.55 & $\mathrm{~N} / \mathrm{A}$ & 3.08 \\
\hline N/A & 4.66 & 9530002K18Rik & 3.54 & A630023P12Rik & 3.08 \\
\hline Gm7750 & 4.64 & 1700008F21Rik & 3.54 & Alox12b & 3.07 \\
\hline N/A & 4.61 & Grhl3 & 3.54 & Hsd3b4 & 3.07 \\
\hline N/A & 4.61 & Smc2 & 3.54 & Caskin1 & 3.07 \\
\hline Gm8026 & 4.61 & Fam46d & 3.54 & Ank3 & 3.07 \\
\hline 4933407C03Rik & 4.61 & Mypop & 3.54 & Helz & 3.06 \\
\hline Tmub2 & 4.59 & Spats2 & 3.53 & Taar7b & 3.06 \\
\hline Tnfrsf 25 & 4.59 & Mpa21 & 3.53 & Gm3602 & 3.06 \\
\hline Gm3269 & 4.57 & Nosip & 3.53 & Gm10094 & 3.06 \\
\hline Gm8297 & 4.57 & Iigp1 & 3.53 & Ptpn9 & 3.06 \\
\hline 9130230L23Rik & 4.56 & Wdr52 & 3.51 & 1700085B03Rik & 3.06 \\
\hline 4831440E17Rik & 4.55 & 4833442J19Rik & 3.51 & Gm7696 & 3.06 \\
\hline N/A & 4.54 & Tiam1 & 3.51 & 2610002I17Rik & 3.06 \\
\hline Maf & 4.54 & Snapc4 & 3.51 & Cav3 & 3.06 \\
\hline Gm7894 & 4.54 & Dgat2 & 3.51 & Slc4a8 & 3.06 \\
\hline 4932431H17Rik & 4.53 & Saps2 & 3.50 & Cacna2d1 & 3.06 \\
\hline E030046B03Rik & 4.53 & Tasp1 & 3.50 & St3gal4 & 3.05 \\
\hline Gm3264 & 4.51 & 9930013L23Rik & 3.50 & Gm5134 & 3.05 \\
\hline Odz3 & 4.51 & Sectmlb & 3.49 & Plod2 & 3.05 \\
\hline Olfr725 & 4.50 & LOC432958 & 3.49 & Gm2282 & 3.05 \\
\hline Frmd6 & 4.49 & Grik2 & 3.49 & Rpl26 & 3.05 \\
\hline Reck & 4.47 & B230216N24Rik & 3.49 & Ly6g6c & 3.05 \\
\hline Cars2 & 4.47 & Pla1a & 3.49 & Gm3453 & 3.05 \\
\hline Themis & 4.46 & Bex1 & 3.48 & Suox & 3.05 \\
\hline Msh2 & 4.46 & N/A & 3.48 & Emilin3 & 3.05 \\
\hline Olfr 1356 & 4.45 & Slc35d1 & 3.48 & 4931422A03Rik & 3.05 \\
\hline E030010N08Rik & 4.44 & N/A & 3.47 & Airn & 3.05 \\
\hline Ninj2 & 4.44 & Zfp444 & 3.47 & Gm8301 & 3.04 \\
\hline Dennd2c & 4.44 & Kcnab3 & 3.47 & Prss23 & 3.04 \\
\hline LOC100038847 & 4.43 & Gm9893 & 3.47 & Exoc31 & 3.04 \\
\hline Ppp2r3a & 4.42 & Afm & 3.46 & Gm3556 & 3.04 \\
\hline Rsad1 & 4.42 & Tecpr1 & 3.46 & Car12 & 3.04 \\
\hline Nicn1 & 4.41 & Gm7980 & 3.46 & N/A & 3.04 \\
\hline $\mathrm{N} / \mathrm{A}$ & 4.40 & V1rc26 & 3.46 & Ipcef1 & 3.03 \\
\hline Osbpl3 & 4.38 & Pyroxd2 & 3.46 & Gm6160 & 3.03 \\
\hline Duxbl & 4.38 & Myolb & 3.45 & Stk30 & 3.03 \\
\hline Olfr1019 & 4.38 & Gemin5 & 3.45 & Txk & 3.03 \\
\hline Ripk4 & 4.37 & Dzip1 & 3.45 & Klra4 & 3.03 \\
\hline Ermp1 & 4.37 & Pabpc3 & 3.45 & Icos & 3.03 \\
\hline Sfmbt2 & 4.33 & Olfr781 & 3.45 & Ciapin1 & 3.02 \\
\hline Gpt2 & 4.33 & Agrn & 3.44 & Frmd4b & 3.02 \\
\hline Myct1 & 4.32 & Fam98c & 3.44 & Gm3278 & 3.02 \\
\hline E330026B02Rik & 4.31 & Fam65a & 3.44 & Scrn3 & 3.02 \\
\hline
\end{tabular}




\begin{tabular}{|c|c|c|c|c|c|}
\hline Zbtb16 & 4.31 & Plekhg1 & 3.44 & 0610031O16Rik & 3.02 \\
\hline N/A & 4.29 & Pbld & 3.44 & Brwd2 & 3.02 \\
\hline 2010005H15Rik & 4.29 & Epb4.111 & 3.43 & Numbl & 3.02 \\
\hline Rragd & 4.28 & Zap70 & 3.43 & Raph1 & 3.02 \\
\hline Ephb3 & 4.28 & Kcnk13 & 3.43 & $\mathrm{~N} / \mathrm{A}$ & 3.01 \\
\hline Treh & 4.28 & Mrgprh & 3.43 & N/A & 3.01 \\
\hline Krt72 & 4.28 & Gm8519 & 3.42 & Klrg1 & 3.01 \\
\hline Snx 16 & 4.28 & Cntfr & 3.42 & Srd5al & 3.01 \\
\hline Tox & 4.28 & $\mathrm{~N} / \mathrm{A}$ & 3.41 & & \\
\hline Il2ra & 25.65 & Atxn711 & 4.27 & Trim37 & 3.41 \\
\hline N/A & 18.99 & Gm5282 & 4.25 & Ksr2 & 3.41 \\
\hline Gm9119 & 15.47 & St3gal3 & 4.25 & ENSMUSG00000079376 & 3.41 \\
\hline II2ra & 15.05 & 4930417O13Rik & 4.24 & Ptpn5 & 3.40 \\
\hline N/A & 14.55 & Trerf1 & 4.24 & 9230117E06Rik & 3.40 \\
\hline Ctla4 & 14.24 & Klk6 & 4.23 & N/A & 3.40 \\
\hline Gal3st1 & 12.37 & 2610042L04Rik & 4.22 & N/A & 3.40 \\
\hline Gm3453 & 12.21 & Cyp4f41-ps & 4.22 & Plekha1 & 3.39 \\
\hline Gal & 12.20 & Clen1 & 4.21 & Trav3n-3 & 3.39 \\
\hline ENSMUSG00000072735 & 11.93 & Abcb7 & 4.20 & Lrsam1 & 3.39 \\
\hline Foxp3 & 11.69 & Bcs11 & 4.20 & Olfr109 & 3.39 \\
\hline Cyb5r2 & 11.65 & Stk19 & 4.18 & Rsbn1 & 3.39 \\
\hline Phkg1 & 10.53 & Sectm1a & 4.18 & Odf1 & 3.39 \\
\hline Ikzf2 & 10.44 & Fmrlnb & 4.17 & $\mathrm{Mc} 2 \mathrm{r}$ & 3.38 \\
\hline Evc2 & 10.17 & Pnkd & 4.17 & Ifna6 & 3.38 \\
\hline $\mathrm{Il1} 7 \mathrm{rc}$ & 10.00 & N/A & 4.17 & Gm7223 & 3.38 \\
\hline Plekhg5 & 9.93 & Gpr110 & 4.17 & Cntn4 & 3.38 \\
\hline ENSMUSG00000072735 & 9.66 & Inpp $4 b$ & 4.17 & N/A & 3.38 \\
\hline Acer2 & 9.56 & Gatsl3 & 4.17 & Gm10228 & 3.38 \\
\hline $\mathrm{Neb}$ & 9.55 & Dapk1 & 4.16 & Gm5169 & 3.37 \\
\hline Gpr45 & 9.49 & Gm3455 & 4.15 & R3hcc1 & 3.37 \\
\hline D15Wsu169e & 9.47 & Gm14717 & 4.14 & Slc38a1 & 3.37 \\
\hline Brunol5 & 9.44 & 1700001E04Rik & 4.14 & Inpp4b & 3.37 \\
\hline Pxdn & 9.44 & Pde4a & 4.13 & Nphp3 & 3.37 \\
\hline Gpr83 & 9.43 & Slc35f2 & 4.13 & Csnk1g1 & 3.36 \\
\hline ENSMUSG00000072735 & 9.43 & Adam6b & 4.13 & Jazf1 & 3.36 \\
\hline Gm3727 & 9.36 & Penk & 4.13 & Arhgdig & 3.36 \\
\hline Gm3727 & 9.25 & 2510048L02Rik & 4.13 & Etaal & 3.36 \\
\hline N/A & 9.24 & Casp3 & 4.12 & $\mathrm{Cul} 2$ & 3.36 \\
\hline Gm11744 & 9.05 & Dcaf17 & 4.12 & Gm10837 & 3.36 \\
\hline Gm3339 & 8.66 & Gm3182 & 4.12 & Ppp2r3a & 3.36 \\
\hline Dpy1912 & 8.60 & 1500015O10Rik & 4.11 & Gm1574 & 3.35 \\
\hline Caskin2 & 8.31 & Acsl4 & 4.11 & Tspan12 & 3.35 \\
\hline Ikzf2 & 8.22 & Ddx43 & 4.10 & Magi3 & 3.35 \\
\hline Tubgcp5 & 8.17 & AI987944 & 4.09 & 1110059M19Rik & 3.35 \\
\hline Gm2974 & 8.16 & Plin1 & 4.09 & Cpsf4l & 3.34 \\
\hline C230088H06Rik & 8.08 & Tox & 4.09 & Parp4 & 3.34 \\
\hline Fbxw27 & 8.05 & Gm10338 & 4.07 & Galr3 & 3.34 \\
\hline Gm14005 & 8.04 & Zscan12 & 4.06 & Adam33 & 3.34 \\
\hline
\end{tabular}




\begin{tabular}{|c|c|c|c|c|c|}
\hline Gm8362 & 7.95 & Fam71e1 & 4.06 & Frs3 & 3.33 \\
\hline Gm8297 & 7.93 & $\mathrm{Neb}$ & 4.06 & Ptgdr & 3.33 \\
\hline Pla2g2d & 7.93 & 100039441 & 4.05 & BE691133 & 3.33 \\
\hline Slc22a12 & 7.91 & BC106179 & 4.05 & Brp441 & 3.33 \\
\hline N/A & 7.87 & N/A & 4.05 & Gm11468 & 3.33 \\
\hline Cadm3 & 7.81 & Stab1 & 4.04 & Detn4 & 3.33 \\
\hline Cyhr1 & 7.58 & Tnfsf13b & 4.04 & E330021D16Rik & 3.33 \\
\hline B630019K06Rik & 7.58 & Mdfi & 4.03 & Gm3764 & 3.32 \\
\hline Inpp4b & 7.50 & A930002C04Rik & 4.03 & $\mathrm{Cd} 300 \lg$ & 3.32 \\
\hline Ctla4 & 7.49 & Slc23a3 & 4.03 & Atg2a & 3.32 \\
\hline Сур2u1 & 7.47 & Col6a3 & 4.02 & Ankrd9 & 3.32 \\
\hline Gm3182 & 7.44 & Ghrh & 4.01 & $\mathrm{Gm} 7225$ & 3.32 \\
\hline Tgfb2 & 7.43 & A930017M01Rik & 4.01 & Pnpla7 & 3.32 \\
\hline Vwce & 7.41 & Itih51 & 4.01 & $\mathrm{Cd} 96$ & 3.31 \\
\hline LOC100036568 & 7.32 & Aurkc & 4.00 & 4833422F24Rik & 3.31 \\
\hline 1700029I01Rik & 7.31 & Itga6 & 4.00 & Thnsl2 & 3.31 \\
\hline Olfr701 & 7.29 & Mfrp & 3.99 & Pdcd11 & 3.31 \\
\hline Rfc3 & 7.29 & 1700042G15Rik & 3.99 & Robo4 & 3.31 \\
\hline Gm10014 & 7.22 & Mageh1 & 3.98 & Aven & 3.31 \\
\hline N/A & 7.20 & Ptpn13 & 3.98 & 1700026L06Rik & 3.31 \\
\hline LOC100038847 & 7.16 & Olfr227 & 3.98 & Lrig2 & 3.31 \\
\hline 544988 & 7.09 & 1700028M03Rik & 3.98 & Ehbp1 & 3.31 \\
\hline Gm4489 & 7.07 & Gpatch4 & 3.98 & Kctd 9 & 3.30 \\
\hline LOC100038847 & 6.95 & Pxmp2 & 3.97 & Zbtb37 & 3.30 \\
\hline Nlrx1 & 6.94 & Mllt3 & 3.97 & Lrrc34 & 3.30 \\
\hline N/A & 6.92 & Gm10250 & 3.97 & Zfp30 & 3.30 \\
\hline Gm3642 & 6.92 & Cux1 & 3.96 & Ano2 & 3.29 \\
\hline $\operatorname{Tgm} 1$ & 6.90 & Csmd1 & 3.96 & N/A & 3.29 \\
\hline Dmd & 6.88 & Ptger3 & 3.96 & Tmem134 & 3.29 \\
\hline Foxp3 & 6.85 & Gm3990 & 3.95 & Sh2d6 & 3.29 \\
\hline ENSMUSG00000072735 & 6.82 & 2010005J08Rik & 3.94 & Olfr78 & 3.29 \\
\hline Grial & 6.82 & Olfr623 & 3.94 & Mapk8 & 3.29 \\
\hline Arhgef15 & 6.81 & ENSMUSG00000072735 & 3.94 & Upp1 & 3.29 \\
\hline Gm2888 & 6.79 & March7 & 3.94 & $\mathrm{Gm} 2046$ & 3.29 \\
\hline Fdft1 & 6.73 & $\mathrm{~N} / \mathrm{A}$ & 3.94 & Tex21 & 3.28 \\
\hline Gm3642 & 6.72 & Slc9a3 & 3.93 & Tnfrsf4 & 3.28 \\
\hline Nck2 & 6.70 & Rbm9 & 3.93 & Nol11 & 3.28 \\
\hline Adamts14 & 6.64 & Dtwd1 & 3.93 & 1700092C10Rik & 3.28 \\
\hline Zfp142 & 6.60 & $\mathrm{C} 77370$ & 3.93 & Gm3916 & 3.28 \\
\hline Gm3269 & 6.59 & N/A & 3.92 & Dmxl2 & 3.28 \\
\hline Gm3411 & 6.56 & Fbxw13 & 3.92 & ENSMUSG00000072735 & 3.28 \\
\hline 544988 & 6.53 & Amz2 & 3.92 & ENSMUSG00000079376 & 3.27 \\
\hline 9630058J23Rik & 6.53 & Nsl1 & 3.92 & 4930587E11Rik & 3.27 \\
\hline 2010109N18Rik & 6.51 & Plxna3 & 3.92 & Plcll & 3.27 \\
\hline N/A & 6.51 & Ppme1 & 3.90 & Srgap3 & 3.27 \\
\hline Brap & 6.51 & Gcgr & 3.90 & Prss39 & 3.27 \\
\hline Tmem 210 & 6.47 & Sgcd & 3.90 & Dapk3 & 3.26 \\
\hline
\end{tabular}




\begin{tabular}{|c|c|c|c|c|c|}
\hline 4930486G11Rik & 6.46 & N/A & 3.89 & Fbxw24 & 3.26 \\
\hline Vmn2r46 & 6.46 & ENSMUSG00000068790 & 3.89 & Gm3626 & 3.26 \\
\hline 1110017D15Rik & 6.46 & Olfr658 & 3.88 & Mtap4 & 3.26 \\
\hline N/A & 6.43 & Fbxo15 & 3.88 & Gm3253 & 3.25 \\
\hline N/A & 6.43 & Mrgprb4 & 3.88 & Cypt6 & 3.25 \\
\hline N/A & 6.38 & Ncoa7 & 3.87 & Aatf & 3.25 \\
\hline Gm3518 & 6.36 & Grin1 & 3.87 & Il2rb & 3.25 \\
\hline C430002E04Rik & 6.26 & 4933400A11Rik & 3.87 & Fam160a1 & 3.24 \\
\hline Gm13620 & 6.23 & Vmn2r72 & 3.87 & Ece1 & 3.24 \\
\hline Gm3685 & 6.23 & Pfkp & 3.87 & Nkx2-6 & 3.24 \\
\hline Zscan 10 & 6.22 & Igl & 3.86 & Pik3r2 & 3.24 \\
\hline Gm10340 & 6.22 & 4930445K14Rik & 3.86 & Slc25a21 & 3.24 \\
\hline Gm3159 & 6.21 & Krtap5-4 & 3.85 & Ptgfrn & 3.24 \\
\hline 5830403L16Rik & 6.16 & Gm3424 & 3.85 & N/A & 3.24 \\
\hline Gm3127 & 6.15 & $\mathrm{Cd} 247$ & 3.85 & Tbcel & 3.23 \\
\hline B930046C15Rik & 6.13 & Samsn1 & 3.85 & Sgip1 & 3.23 \\
\hline Syne2 & 6.10 & Uty & 3.84 & 1700023L04Rik & 3.23 \\
\hline Gm3029 & 6.10 & Galk1 & 3.84 & Catsper3 & 3.23 \\
\hline 1600002D24Rik & 6.06 & 1700029G01Rik & 3.84 & Dgka & 3.23 \\
\hline Gm2224 & 6.04 & Agrn & 3.83 & 4930433N12Rik & 3.23 \\
\hline Gm4801 & 6.00 & Lrig2 & 3.83 & Rdh16 & 3.22 \\
\hline N/A & 5.97 & Slc25a27 & 3.83 & BC011248 & 3.22 \\
\hline Pitpnc1 & 5.94 & Gjb4 & 3.83 & Dlgap1 & 3.22 \\
\hline Gm3476 & 5.94 & Rgs16 & 3.83 & Olfr1283 & 3.22 \\
\hline 6430562O15Rik & 5.92 & Cntn1 & 3.82 & Osbpl3 & 3.22 \\
\hline Vmn2r66 & 5.92 & Fndc7 & 3.82 & Foxi2 & 3.21 \\
\hline Gm3029 & 5.91 & Itk & 3.82 & Fam186a & 3.20 \\
\hline Gm3115 & 5.89 & N/A & 3.82 & Gm8356 & 3.20 \\
\hline Ndp & 5.84 & $\mathrm{H} 1 \mathrm{fx}$ & 3.81 & Timp4 & 3.20 \\
\hline Zfp329 & 5.83 & Pik3c2a & 3.80 & Tbc1d8 & 3.20 \\
\hline Gpr64 & 5.82 & Ctsj & 3.80 & Srd5a1 & 3.20 \\
\hline Nav2 & 5.81 & Emid1 & 3.80 & Olfr242 & 3.20 \\
\hline Aven & 5.80 & Serpinale & 3.79 & Sel11 & 3.19 \\
\hline D030054H15Rik & 5.79 & Gm1330 & 3.79 & Mbnl2 & 3.19 \\
\hline Grik5 & 5.76 & Tbcld4 & 3.79 & Plac9 & 3.19 \\
\hline Sgsm3 & 5.71 & Hs1bp3 & 3.79 & N/A & 3.19 \\
\hline Ovol2 & 5.70 & Olfr961 & 3.79 & Slc12a1 & 3.19 \\
\hline Mc1r & 5.65 & Pask & 3.78 & Zfp169 & 3.19 \\
\hline Gm10371 & 5.65 & ВC060267 & 3.78 & Dok7 & 3.18 \\
\hline N/A & 5.62 & Kpna1 & 3.78 & Gm2275 & 3.18 \\
\hline Luzp2 & 5.60 & Arg1 & 3.77 & Gm2643 & 3.18 \\
\hline Pthlh & 5.59 & Cts8 & 3.77 & Dpep2 & 3.18 \\
\hline 1700021F07Rik & 5.58 & Suclg1 & 3.77 & Pard6b & 3.18 \\
\hline Ccbe 1 & 5.56 & 1700001E04Rik & 3.77 & Cyp17a1 & 3.17 \\
\hline Cul7 & 5.55 & Synpo2 & 3.77 & 9330111N05Rik & 3.17 \\
\hline Cpped1 & 5.55 & 6030458C11Rik & 3.77 & Ccdc33 & 3.17 \\
\hline Fmnl3 & 5.54 & 1190002H23Rik & 3.77 & Tub & 3.17 \\
\hline D6Wsu163e & 5.53 & Rpusd3 & 3.76 & Rp1711 & 3.17 \\
\hline
\end{tabular}




\begin{tabular}{|c|c|c|c|c|c|}
\hline Serpinb9d & 5.49 & Gm6710 & 3.76 & AW495222 & 3.17 \\
\hline E030025P04Rik & 5.49 & Ikbkap & 3.76 & Ipcef1 & 3.17 \\
\hline Skap1 & 5.49 & N/A & 3.76 & Tle2 & 3.17 \\
\hline D0H4S114 & 5.47 & 4921523L03Rik & 3.75 & B3galnt2 & 3.17 \\
\hline Piwil2 & 5.45 & Speer4f & 3.75 & Ndel1 & 3.16 \\
\hline 4930524L23Rik & 5.45 & Gm3047 & 3.75 & Atp6v1c2 & 3.16 \\
\hline Ykt6 & 5.43 & Synpr & 3.74 & Hnrpll & 3.16 \\
\hline S1c24a3 & 5.40 & $\mathrm{~N} / \mathrm{A}$ & 3.73 & Prrg1 & 3.16 \\
\hline N/A & 5.39 & 9030624G23Rik & 3.72 & Cyp2j13 & 3.16 \\
\hline Gm6337 & 5.38 & Trp53inp2 & 3.72 & Espn & 3.16 \\
\hline Gm3149 & 5.34 & 9130401M01Rik & 3.72 & Mup1 & 3.15 \\
\hline Tnfrsf9 & 5.29 & Myst4 & 3.72 & Ptprr & 3.15 \\
\hline Ttn & 5.28 & Gm12836 & 3.72 & Snx11 & 3.15 \\
\hline Gpr52 & 5.27 & 2810039B14Rik & 3.71 & Chchd8 & 3.15 \\
\hline Cntfr & 5.26 & Fastk & 3.71 & Dnm1 & 3.15 \\
\hline ENSMUSG00000079376 & 5.25 & Inpp4b & 3.71 & Tbc1d25 & 3.15 \\
\hline LOC100038847 & 5.22 & N/A & 3.70 & Olfr 1120 & 3.14 \\
\hline Mybpc2 & 5.22 & Prlh & 3.70 & Gm3981 & 3.14 \\
\hline Cdon & 5.16 & Mcm8 & 3.70 & Morc2a & 3.14 \\
\hline Slitrk6 & 5.16 & Gm15340 & 3.70 & Ttll7 & 3.14 \\
\hline Dom3z & 5.14 & Gm4926 & 3.69 & Irf6 & 3.14 \\
\hline Gm3149 & 5.14 & Ebpl & 3.69 & A830039H05Rik & 3.13 \\
\hline Gm16521 & 5.14 & N/A & 3.69 & 1700024B18Rik & 3.13 \\
\hline Smarcal1 & 5.14 & 4930417O13Rik & 3.68 & Trapla & 3.13 \\
\hline C230099D08Rik & 5.13 & Pcdh15 & 3.68 & Vmn2r10 & 3.13 \\
\hline Olfr1252 & 5.11 & Ctla4 & 3.68 & Nrn1 & 3.13 \\
\hline 4930599N23Rik & 5.11 & 4933432I09Rik & 3.68 & Mapkapk3 & 3.13 \\
\hline Gm3642 & 5.10 & Hsd17b2 & 3.68 & 3110082J24Rik & 3.13 \\
\hline Gm5634 & 5.09 & Fbp1 & 3.67 & Ccdc65 & 3.13 \\
\hline Cngb1 & 5.08 & Gm5795 & 3.66 & Spag6 & 3.13 \\
\hline Pax3 & 5.07 & Gm8159 & 3.66 & AI428936 & 3.12 \\
\hline ENSMUSG00000068790 & 5.07 & Atf7 & 3.66 & Tiam1 & 3.12 \\
\hline 4632404H12Rik & 5.05 & $\mathrm{Kdm} 4 \mathrm{a}$ & 3.66 & Cenpk & 3.12 \\
\hline Vill & 5.03 & Ocrl & 3.66 & Rapsn & 3.12 \\
\hline Gm8050 & 5.02 & Sgoll & 3.65 & $\operatorname{Tm} 2 \mathrm{~d} 3$ & 3.11 \\
\hline Anks $4 b$ & 5.00 & Prox2 & 3.65 & Tiam1 & 3.11 \\
\hline St3gal6 & 4.96 & Rnf26 & 3.65 & Tle2 & 3.11 \\
\hline 1700034I23Rik & 4.96 & N/A & 3.64 & Wbp11 & 3.11 \\
\hline $\mathrm{Gm} 3172$ & 4.95 & Bub1 & 3.64 & Olfr 1128 & 3.11 \\
\hline Spata18 & 4.93 & Trim63 & 3.63 & Art1 & 3.11 \\
\hline Plcg1 & 4.92 & Slc6a9 & 3.62 & Grin3a & 3.11 \\
\hline Has2as & 4.91 & Dst & 3.61 & 1700110K17Rik & 3.10 \\
\hline $\mathrm{Ntn} 4$ & 4.90 & AI428936 & 3.61 & Bcat1 & 3.10 \\
\hline Skap1 & 4.88 & Marveld2 & 3.60 & Iigp1 & 3.10 \\
\hline Сyp2c50 & 4.88 & Esrrb & 3.60 & $\mathrm{Pla} 2 \mathrm{~g} 4 \mathrm{e}$ & 3.10 \\
\hline Cope & 4.87 & Gm4699 & 3.60 & Rpusd1 & 3.10 \\
\hline N/A & 4.86 & Ttyh1 & 3.59 & Olfr638 & 3.10 \\
\hline Gm3642 & 4.86 & Rgs16 & 3.59 & Agbl2 & 3.10 \\
\hline
\end{tabular}

PeerJ reviewing PDF | (v2014:07:2417:1:2:NEW 19 Aug 2014) 


\begin{tabular}{|c|c|c|c|c|c|}
\hline Tnfrsf18 & 4.85 & 8030463A06Rik & 3.59 & 4921509O09Rik & 3.09 \\
\hline N/A & 4.82 & 4930578E11Rik & 3.58 & Olfr389 & 3.09 \\
\hline Snhg11 & 4.81 & Cacnb2 & 3.57 & Pcsk4 & 3.09 \\
\hline Gm6121 & 4.81 & Setd3 & 3.57 & Pou2f1 & 3.09 \\
\hline Ncoa7 & 4.80 & N/A & 3.57 & Brec3 & 3.09 \\
\hline 1700025M24Rik & 4.75 & Gm2957 & 3.56 & Gm3034 & 3.09 \\
\hline S100a7a & 4.75 & Magea3 & 3.56 & Gm8362 & 3.09 \\
\hline Olfr140 & 4.74 & Syngr3 & 3.56 & D030028A08Rik & 3.09 \\
\hline Crem & 4.73 & Gm3127 & 3.55 & Fam118b & 3.08 \\
\hline Gab3 & 4.72 & ENSMUSG00000068790 & 3.55 & Ccdc126 & 3.08 \\
\hline Ift80 & 4.71 & Tmem176a & 3.55 & Fbxw4 & 3.08 \\
\hline Secisbp2 & 4.69 & 1700081N11Rik & 3.55 & Cish & 3.08 \\
\hline 1110019B22Rik & 4.67 & N/A & 3.55 & N/A & 3.08 \\
\hline $\mathrm{N} / \mathrm{A}$ & 4.66 & 9530002K18Rik & 3.54 & A630023P12Rik & 3.08 \\
\hline Gm7750 & 4.64 & 1700008F21Rik & 3.54 & Alox12b & 3.07 \\
\hline N/A & 4.61 & Grhl3 & 3.54 & Hsd $3 b 4$ & 3.07 \\
\hline N/A & 4.61 & Smc2 & 3.54 & Caskin1 & 3.07 \\
\hline Gm8026 & 4.61 & Fam $46 \mathrm{~d}$ & 3.54 & Ank3 & 3.07 \\
\hline 4933407C03Rik & 4.61 & Мyрор & 3.54 & Helz & 3.06 \\
\hline Tmub2 & 4.59 & Spats2 & 3.53 & Taar7b & 3.06 \\
\hline Tnfrsf 25 & 4.59 & Mpa21 & 3.53 & Gm3602 & 3.06 \\
\hline Gm3269 & 4.57 & Nosip & 3.53 & Gm10094 & 3.06 \\
\hline Gm8297 & 4.57 & Iigp1 & 3.53 & Ptpn9 & 3.06 \\
\hline 9130230L23Rik & 4.56 & Wdr52 & 3.51 & 1700085B03Rik & 3.06 \\
\hline 4831440E17Rik & 4.55 & 4833442J19Rik & 3.51 & Gm7696 & 3.06 \\
\hline $\mathrm{N} / \mathrm{A}$ & 4.54 & Tiam1 & 3.51 & 2610002I17Rik & 3.06 \\
\hline Maf & 4.54 & Snapc4 & 3.51 & Cav3 & 3.06 \\
\hline Gm7894 & 4.54 & Dgat2 & 3.51 & Slc4a8 & 3.06 \\
\hline 4932431H17Rik & 4.53 & Saps2 & 3.50 & Cacna2d1 & 3.06 \\
\hline E030046B03Rik & 4.53 & Tasp1 & 3.50 & St3gal4 & 3.05 \\
\hline Gm3264 & 4.51 & 9930013L23Rik & 3.50 & Gm5134 & 3.05 \\
\hline Odz3 & 4.51 & Sectm1b & 3.49 & Plod2 & 3.05 \\
\hline Olfr725 & 4.50 & LOC432958 & 3.49 & Gm2282 & 3.05 \\
\hline Frmd6 & 4.49 & Grik2 & 3.49 & Rpl26 & 3.05 \\
\hline Reck & 4.47 & B230216N24Rik & 3.49 & Ly6g6c & 3.05 \\
\hline Cars2 & 4.47 & Pla1a & 3.49 & Gm3453 & 3.05 \\
\hline Themis & 4.46 & Bex1 & 3.48 & Suox & 3.05 \\
\hline Msh2 & 4.46 & N/A & 3.48 & Emilin3 & 3.05 \\
\hline Olfr1356 & 4.45 & Slc35d1 & 3.48 & 4931422A03Rik & 3.05 \\
\hline E030010N08Rik & 4.44 & N/A & 3.47 & Airn & 3.05 \\
\hline Ninj2 & 4.44 & Zfp444 & 3.47 & Gm8301 & 3.04 \\
\hline Dennd2c & 4.44 & Kcnab3 & 3.47 & $\operatorname{Prss} 23$ & 3.04 \\
\hline LOC100038847 & 4.43 & Gm9893 & 3.47 & Exoc31 & 3.04 \\
\hline Ppp2r3a & 4.42 & Afm & 3.46 & Gm3556 & 3.04 \\
\hline Rsad1 & 4.42 & Tecpr1 & 3.46 & Car12 & 3.04 \\
\hline Nicn1 & 4.41 & Gm7980 & 3.46 & N/A & 3.04 \\
\hline N/A & 4.40 & V1rc26 & 3.46 & Ipcef1 & 3.03 \\
\hline Osbpl3 & 4.38 & Pyroxd2 & 3.46 & Gm6160 & 3.03 \\
\hline
\end{tabular}




\begin{tabular}{|c|c|c|c|c|c|}
\hline Duxbl & 4.38 & Myolb & 3.45 & Stk30 & 3.03 \\
\hline Olfr1019 & 4.38 & Gemin 5 & 3.45 & Txk & 3.03 \\
\hline Ripk4 & 4.37 & Dzip1 & 3.45 & Klra4 & 3.03 \\
\hline Ermp1 & 4.37 & Pabpc3 & 3.45 & Icos & 3.03 \\
\hline Sfmbt2 & 4.33 & Olfr781 & 3.45 & Ciapin1 & 3.02 \\
\hline Gpt2 & 4.33 & Agrn & 3.44 & Frmd4b & 3.02 \\
\hline Myct1 & 4.32 & Fam98c & 3.44 & Gm3278 & 3.02 \\
\hline E330026B02Rik & 4.31 & Fam65a & 3.44 & Scrn3 & 3.02 \\
\hline Zbtb16 & 4.31 & Plekhg1 & 3.44 & 0610031O16Rik & 3.02 \\
\hline $\mathrm{N} / \mathrm{A}$ & 4.29 & Pbld & 3.44 & Brwd2 & 3.02 \\
\hline 2010005H15Rik & 4.29 & Epb4.111 & 3.43 & Numbl & 3.02 \\
\hline Rragd & 4.28 & Zap70 & 3.43 & Raph1 & 3.02 \\
\hline Ephb3 & 4.28 & Kcnk13 & 3.43 & N/A & 3.01 \\
\hline Treh & 4.28 & Mrgprh & 3.43 & N/A & 3.01 \\
\hline Krt72 & 4.28 & Gm8519 & 3.42 & Klrg1 & 3.01 \\
\hline Snx 16 & 4.28 & Cntfr & 3.42 & Srd5a1 & 3.01 \\
\hline Tox & 4.28 & N/A & 3.41 & & \\
\hline
\end{tabular}




\section{Table 4(on next page) \\ KEGG pathways annotation of abnormal miRNA target genes}

1. Gray indicated downregulated target genes in KEGG pathway.

2. In differentially expressed genes, 15 miRNA target genes were enriched into $\mathrm{T}$ cell receptor (TCR) signaling pathway (Figure 3). 


\begin{tabular}{|c|c|c|}
\hline Pathway & MAPP name & Enrichment Score \\
\hline mmu00562 & Inositol phosphate metabolism & 3.988221 \\
\hline mmu04070 & Phosphatidylinositol signaling system & 3.533671 \\
\hline mmu05410 & Hypertrophic cardiomyopathy (HCM) & 2.394271 \\
\hline mmu04725 & Cholinergic synapse & 2.227839 \\
\hline mmu05412 & Arrhythmogenic right ventricular cardiomyopathy (ARVC) & 2.126784 \\
\hline mmu04724 & Glutamatergic synapse & 2.109772 \\
\hline mmu03460 & Fanconi anemia pathway & 2.017738 \\
\hline mmu05142 & Chagas disease (American trypanosomiasis) & 2.010757 \\
\hline $\mathrm{mmu} 04150$ & mTOR signaling pathway & 1.906663 \\
\hline mmu04660 & $\mathrm{T}$ cell receptor signaling pathway & 1.713143 \\
\hline mmu05322 & Systemic lupus erythematosus & 12.6937 \\
\hline mmu04640 & Hematopoietic cell lineage & 6.723747 \\
\hline mmu05034 & Alcoholism & 6.20107 \\
\hline mmu05152 & Tuberculosis & 5.152889 \\
\hline mmu04662 & B cell receptor signaling pathway & 4.675411 \\
\hline mmu05202 & Transcriptional misregulation in cancer & 4.643977 \\
\hline mmu04672 & Intestinal immune network for IgA production & 4.281526 \\
\hline mmu04380 & Osteoclast differentiation & 4.255375 \\
\hline mmu05150 & Staphylococcus aureus infection & 3.867061 \\
\hline mmu05340 & Primary immunodeficiency & 3.857659 \\
\hline
\end{tabular}

1. Gray indicated downregulated target genes in KEGG pathway.

2. In differentially expressed genes, 15 miRNA target genes were enriched into T cell receptor (TCR) signaling pathway (Figure 3). 


\section{Figure 1}

miRNA expression in CCR6+ Tregs

CCR6+Tregs and CCR6-Tregs were purified from splenocytes in Balb/c mice. The expression of miRNAs in cells was analyzed by microarray array. (A) A heat map of miRNA microarray. (B) A pie graph of miRNA distribution. (C) Predication of putative 6 miRNAs associated with potential proliferation activity of CCR6+Tregs based on functional similarity of target sets. 
A

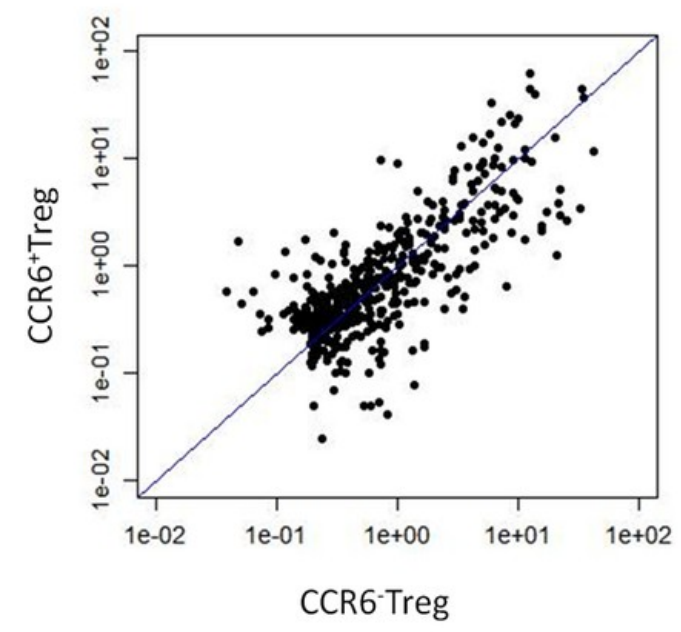

B CCR6 $^{+}$Treg VS.CCR6-Treg Fold-Change
(Normal Control)

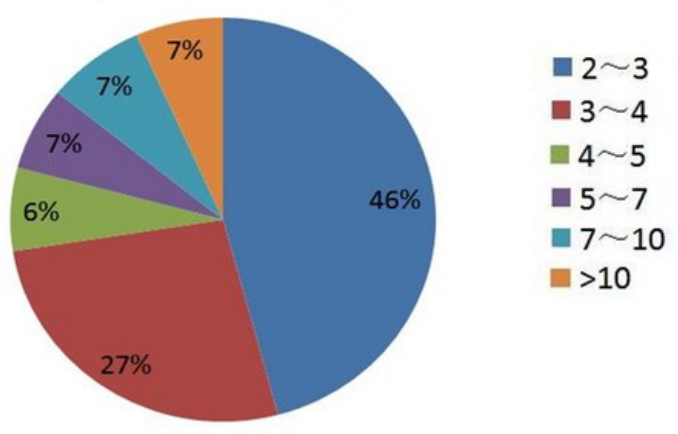

C

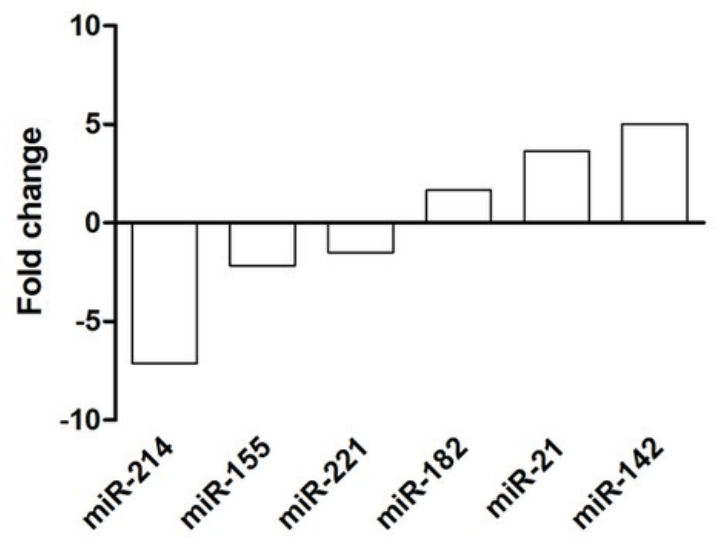




\section{Figure 2}

Gene expression in CCR6+ Tregs detected bymicroarray assays

CCR6+Tregs and CCR6-Tregs were purified from splenocytes in Balb/c mice. The global expression of genes in cells was analyzed by microarray array. (A) A heat map of miRNA microarray. (B) The scatterplot for the variation between CCR6+Tregs and CCR6-Treg. (C). The fold change and frequency between CCR6+Tregs and CCR6-Tregs. 
A

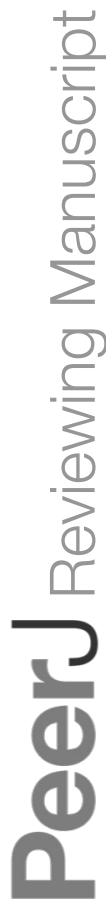

B

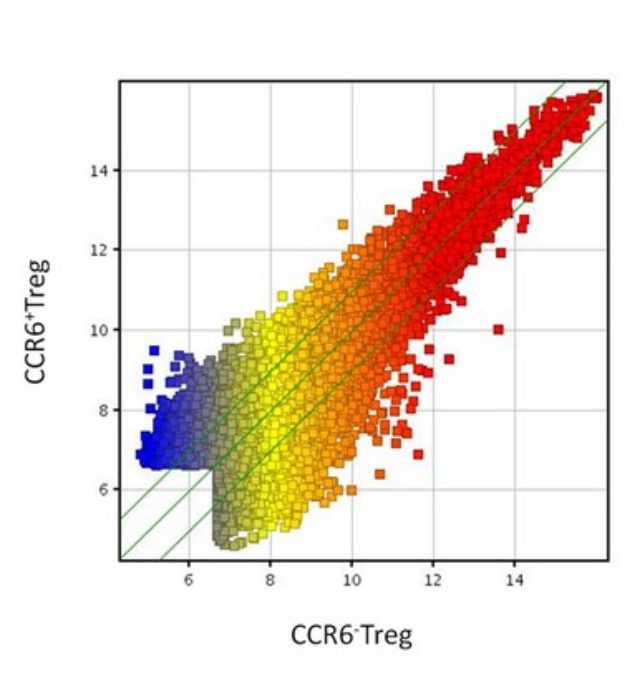

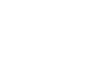

C

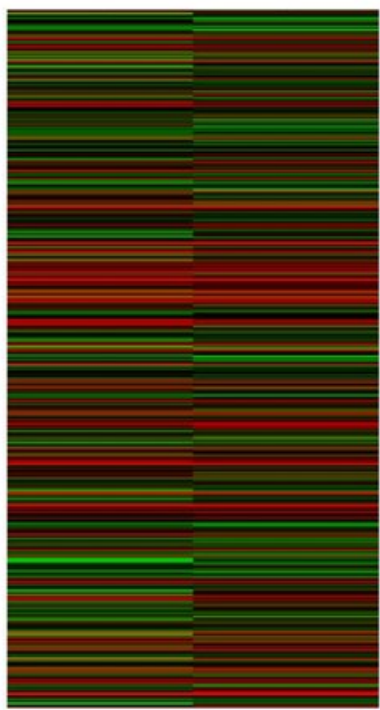

CCR6tres cargeres

mRNA Fold Change

(CCR6 ${ }^{+}$VS.CCR6- Up Regulated)

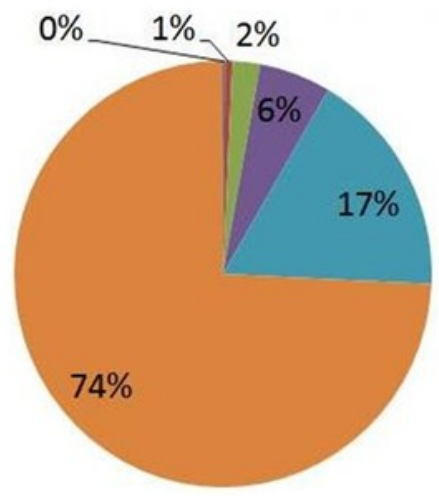




\section{Figure 3}

\section{T cell receptor signaling pathway}
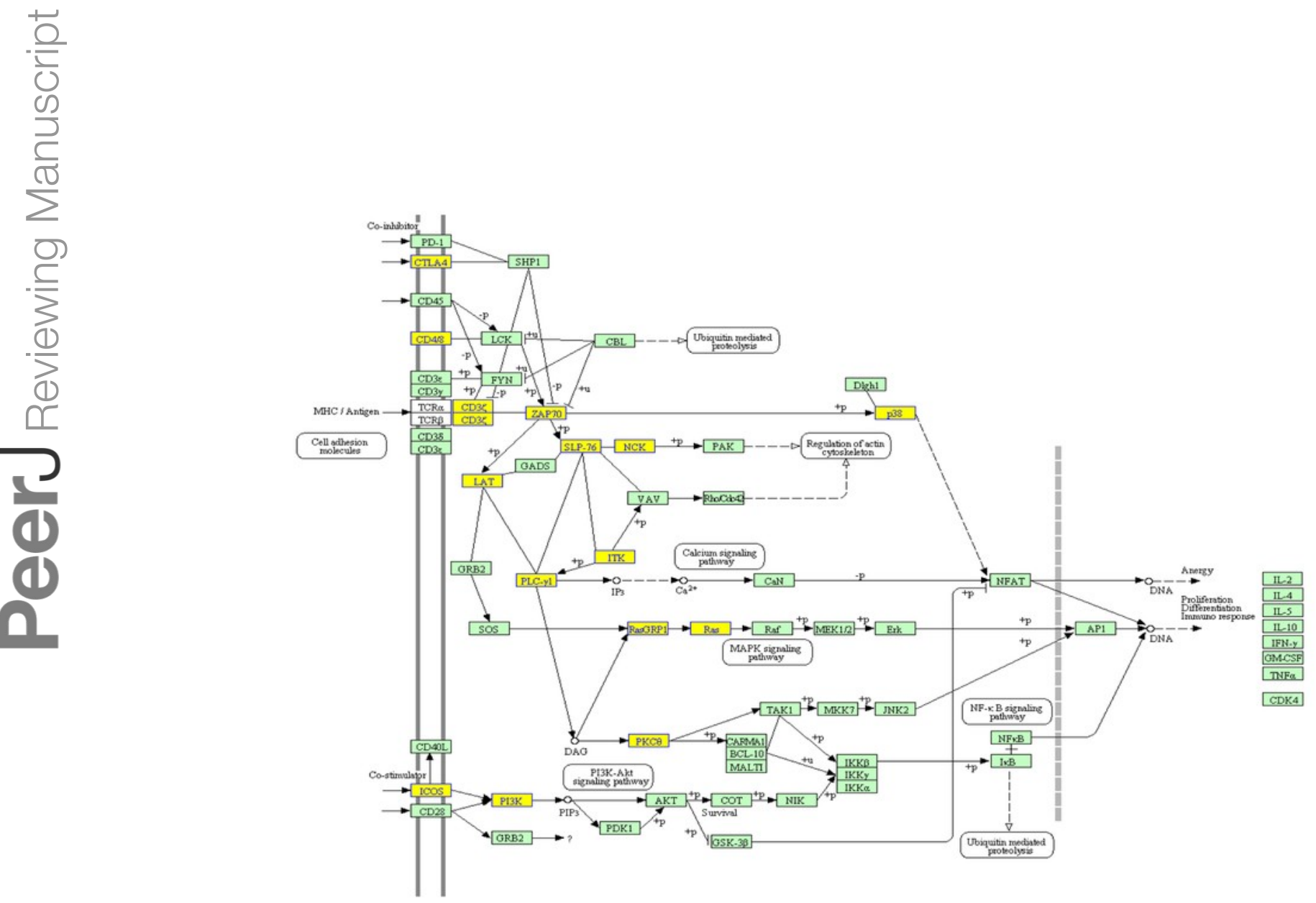